
\title{
$\begin{array}{ll}\text { Research Square } & \begin{array}{l}\text { Preprints are preliminary reports that have not undergone peer review. } \\ \text { They should not be considered conclusive, used to inform clinical practice, } \\ \text { or referenced by the media as validated information. }\end{array}\end{array}$ \\ Self-correcting Sun Compass, Spherical Geometry and Cue-transfers Predict Naïve Migratory Performance
}

James McLaren ( $\nabla$ james.mclaren@uni-oldenburg.de)

University of Oldenburg

Heiko Schmaljohann

Carl von Ossietzky University of Oldenburg

Bernd Blasius

University of Oldenburg https://orcid.org/0000-0002-6558-1462

Article

Keywords: Migratory orientation, naïve migrants, geomagnetic or celestial cues

Posted Date: November 10th, 2021

DOl: https://doi.org/10.21203/rs.3.rs-996110/v1

License: (c) (i) This work is licensed under a Creative Commons Attribution 4.0 International License.

Read Full License

Version of Record: A version of this preprint was published at Communications Biology on October 4th, 2022. See the published version at https://doi.org/10.1038/s42003-022-03995-5. 


\section{Self-correcting sun compass, spherical geometry and cue-transfers predict}

\section{naïve migratory performance}

James D. McLaren ${ }^{1 *}$, Heiko Schmaljohann ${ }^{2,3}$, Bernd Blasius ${ }^{1,4}$

${ }^{1}$ Institute for Chemistry and Biology of the Marine Environment (ICBM), University of

Oldenburg; 26129 Oldenburg, Germany.

${ }^{2}$ Institute for Biology and Environmental Sciences (IBU), Carl von Ossietzky University of

Oldenburg, 26129 Oldenburg, Germany.

${ }^{3}$ Institute of Avian Research, 26386 Wilhelmshaven, Germany.

${ }^{4}$ Helmholtz Institute for Functional Marine Biodiversity (HIFMB), University of Oldenburg;

26129 Oldenburg, Germany.

*Corresponding author. Email: james.mclaren@uol.de 


\begin{abstract}
Migratory orientation of many animals is inheritable, enabling naïve migrants to reach remote destinations independently following stepwise (often, nightly) geomagnetic or celestial cues. Which if any such "compass courses" can explain narrow-front trans-continental routes remains unresolved, and evident error-corrections by naïve migrants remain unexplained. We assessed robustness to errors among airborne compass courses and quantified inaugural migration performance globally, accounting for cue transfers (e.g., sun to star compass), in-flight cue maintenance, and previously-overlooked spherical-geometry (longitude) effects. We found (i) sun-compass courses partially self-correct, making them most robust between flight-steps, (ii) within nocturnal flight-steps, geomagnetic or star-compass headings outperform cue-transferred sun-compass steps, (iii) across diverse airborne migration routes, the relative favourability of sun-compass over other courses increases with increasing goal-area, required flight steps and a spherical-geometry factor. Our results can explain enhanced naïve migrant performance, observed diversity in compass-cue hierarchies, and sun-compass orientation being key to many long-distance inaugural migrations.
\end{abstract}




\section{Introduction}

Seasonal animal migrations have evolved across taxa at spatial scales spanning meters to continents $^{1}$. A critical factor for migratory populations is the ability to perform inaugural ("naïve") migrations without access to a navigational map ${ }^{2,3}$. While migratory routes are often transmitted culturally through collective and social cues ${ }^{4,5}$, many naïve airborne migrants reach population-specific remote destinations (hereafter, goal areas) independently by following innate or inherited headings stepwise relative to proximate geophysical compass cues en route ${ }^{2,6}$. Naïve but migration-ready birds and insects can orient consistently in the horizontal (azimuthal) plane relative to both geomagnetic and celestial directional cues ${ }^{2,6}$. Birds innately distinguish between geomagnetic North and South using geomagnetic inclination (the angle between the geomagnetic field and the horizontal) ${ }^{2,6}$, but celestial compasses accounting for (hourly) rotation in sun azimuth or star patterns need to be learned prior to migration ${ }^{6,7}$. Diagnosis of compass-cues used in-flight and across entire routes remains a major challenge $e^{2,7,8}$. Nonetheless, unassisted naïve migrants probably prioritize one "primary" compass system to determine (e.g., nightly) flight headings, sometimes transferred to a second, in-flight compass. Cue-conflict experiments suggest various contingencies and hierarchies involving “calibration” between compasses, but often prioritization of celestial cues at twilight, particularly among North American migrants ${ }^{6-8}$.

The choice of primary compass can result in substantially different stepwise "compass courses", with five main classes proposed: 1) geographic loxodromes, following constant headings relative to geographic South or North, which is identifiable either by a primary star compass ${ }^{7,9}$ or else by averaging (more reliably available) maximum bands of polarized light at sunrise and sunset ${ }^{10-12}$; 2) geomagnetic loxodromes, following constant headings relative to geomagnetic South or North; 3) gradually-shifting magnetoclinic course, based on maintaining a fixed (transverse) 
projection of proximate geomagnetic inclination en route $\left.{ }^{11,13} ; 4\right)$ fixed (menotactic) sun compass courses, following a constant heading relative to proximate sunrise or sunset azimuth, which naturally shift with date and location $\left.{ }^{11} ; 5\right)$ time-compensated sun compass (TCSC) courses, which can achieve nearly great-circle trajectories due to the "clock shift" induced by crossing longitudes, resulting in increasingly Southward headings (Northward in the Southward Hemisphere $)^{14,15}$. While imprecise stepwise loxodromes based on constant preferred headings can sufficiently explain broad-front migration ${ }^{2,16,17}$, migratory tracking data reveal a diverse picture featuring narrow-front and sharply direction-changing ${ }^{18}$. Indeed, while known birdmigration routes often resemble sun compass and magnetoclinic courses ${ }^{13,15}$, their relative feasibility has been debated ${ }^{11,19}$ and robustness to stepwise errors remains untested. Even more puzzling is the evidence of route-corrections - a hallmark of true navigation ${ }^{2,3}-$ by some naturally and artificially displaced naïve bird migrants en route en-22 $^{2}$

Here, we provide a modelling framework to assess robustness of migratory compass courses across the globe, and identify key geophysical and route-geometric factors governing inaugural migratory performance, here quantified as proportional arrival at goal areas. For simplicity and interpretability, we focused on inaugural airborne migration based on a single inherited or imprinted (initial) heading. We first extended current formulation of compass courses, to account for 1) imprecision within single flight-steps, including possible cue transfers to a second (inflight) compass and in-flight cue maintenance; 2) the effect of stepwise errors on subsequent headings and courses, particularly for the direction-shifting sun-compass and magnetoclinic courses; 3) spherical geometry effects, in particular from the convergence of longitude bands at higher latitudes ${ }^{23}$, which have not been quantified for long-distance animal movements ${ }^{16,18,24,25}$. To assess effects of primary cue-choice, precision and route-geometry in consort, we simulated 
each compass course for both a generic migrant across a broad range of global routes and magnitudes of error, and also for known routes of nine diverse long-distance airborne migratory species, incorporating dynamic geomagnetic data and in-flight error to account for wind or cuerelated drift effects. Finally, we predicted how inaugural migration performance depends on stepwise precision and route-geometric factors, by applying regression and model selection using route-optimized geomagnetic and sun-compass courses among species.

\section{Results}

\section{Between-step and within-step precision}

Table 1 lists terms relating to stepwise movement and geophysical cues, as described in the Methods (equations 1-23). For stepwise compass movement to a goal (equations 1-4, Fig. 1a), the probability of successful arrival (performance) will increase with increasing angular concentration in stepwise headings (von Mises concentration $\kappa$, equation 7), goal-area breadth (ratio of goal radius to migration distance, $R_{\text {goal }} / R_{\text {mig }}$ ) and, following the many-wrongs principle ${ }^{4,26}$, with increasing number of steps, $N$. As a first approximation, assuming independent steps on a plane with a high angular concentration, i.e., small "effective standard error", $\sigma=$ $1 / \sqrt{\kappa}$, migratory performance will follow a cumulative normal distribution as a function of the length-adjusted goal-area breadth, $\beta_{a d j}=\sqrt{N_{0}} R_{\text {goal }} / R_{\text {mig }}$, where $N_{0}$ is the minimum (errorfree) number of flight steps (equations 11-13). 
a

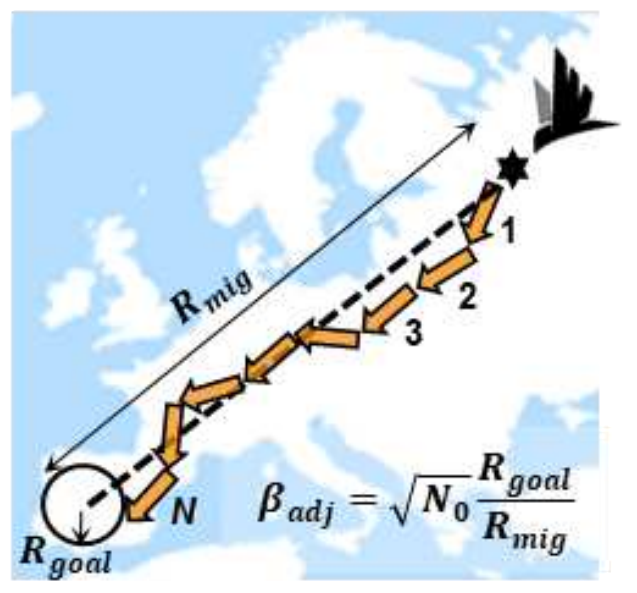

b

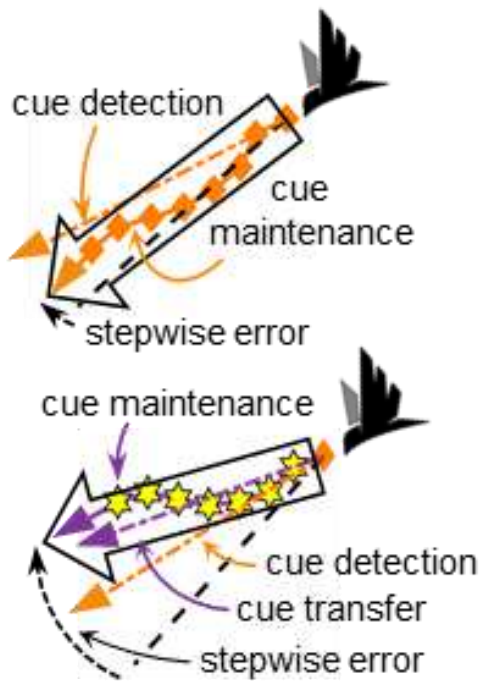

Fig. 1. Stepwise compass movement, between and within-step effects.

(a) Schematic of $N$ migratory steps (orange arrows) based on a single preferred heading (dashed black line) spanning a distance $R_{m i g}$ to a migratory destination or "goal area" (open circle, with radius $\left.R_{\text {goal }}\right)$. For sufficiently small stepwise errors and ignoring spherical geometry effects, the probability of successful arrival is a function of length-adjusted goal-area breadth, $\beta_{a d j}=\sqrt{N_{0}} R_{\text {goal }} / R_{\text {mig }}$, where $N_{0}$ is the minimum (error-free) number of steps (equations 11-13). (b) Within a flight-step based on a single (e.g., geomagnetic) cue, the initial cue-detection error (angle between dashed orange and black lines) can be offset (equation 8a) by in-flight cue maintenance (e.g., re-determined hourly; solid orange line and diamond shapes). (c) Contrastingly, with transfer to a secondary (e.g., star) compass (dashed-purple line), the expected stepwise error will exceed cue-detection errors (equation 8b), regardless of cue maintenance (solid purple line and yellow hexagons). 
Table 1. Definitions of terms describing stepwise movement, within-step precision and

geophysical orientation cues.

\begin{tabular}{|c|c|c|}
\hline & Variable or factor & Description \\
\hline \multirow[t]{4}{*}{$\begin{array}{l}\text { Stepwise } \\
\text { movement }\end{array}$} & Step number, $i$ & $\begin{array}{l}\text { Encompasses departure and (daily or nightly) migratory flights } i= \\
1,2 \ldots, N \text { (Fig. 1a). Subdivided hourly (Fig. 1b-c, Supplemental Fig. 1). }\end{array}$ \\
\hline & Date, $t_{i}$, and hour, $h$ & $\begin{array}{l}\text { Day of year (1-366), and flight hour } h=0, \ldots, n_{H} \text {, with } n_{H} \text { constant per } \\
\text { species (Table 2). Affect geomagnetic }{ }^{27} \text { and sun compass headings }{ }^{11,28}\end{array}$ \\
\hline & Location & $\begin{array}{l}\text { Stepwise latitude, } \varnothing_{i} \text {, and longitude, } \lambda_{i} \text {, in radians (Equations 1-4). } \\
\text { Geomagnetic-dipole simulations use geomagnetic latitude and longitude. }\end{array}$ \\
\hline & Step length, $R_{\text {step }}$ & Stepwise flight distance (radians), here constant per species (Table 2). \\
\hline \multirow{5}{*}{$\begin{array}{l}\text { Geophysical } \\
\text { orientation } \\
\text { cues }\end{array}$} & Geographic axis & Geographic South (S.), geographic North (N.) in the S. Hemisphere. \\
\hline & Geomagnetic axis & $\begin{array}{l}\text { Geomagnetic South, offset from geographic S. by magnetic declination, } \\
\delta_{m} \text { (constant in dipole model, otherwise interpolated from IGRF data }{ }^{27} \text { ). }\end{array}$ \\
\hline & Inclination, $\gamma_{i}$ & Angle of geomagnetic field vector to horizontal. Latitude-dependent ${ }^{13,27}$. \\
\hline & Solar axis & $\begin{array}{l}\text { Sunrise or sunset azimuth (equations 18), possibly time-compensated } \\
\text { between steps (equations } 22-23 \text { ), or alternatively, via maximum band } \\
\text { polarized light }{ }^{10} \text { (perpendicular to sunrise/set azimuth). }\end{array}$ \\
\hline & Stellar axis & Fixed star or centre of rotation, Not time-compensated between steps 9,29 . \\
\hline \multirow[t]{2}{*}{$\begin{array}{l}\text { Orientation } \\
\text { terms }\end{array}$} & $\begin{array}{l}\text { Expected stepwise } \\
\text { heading, } \bar{\alpha}_{i}\end{array}$ & $\begin{array}{l}\text { Clockwise from geographic S. (ccl. from N. in S. Hemisphere), as } \\
\text { determined from primary compass (equations } 14-16,19,22) \text {. Initial } \\
\text { heading inherited or imprinted to geographic heading } \\
\text { 2,6,7. }\end{array}$ \\
\hline & Stepwise error, $\varepsilon_{i}^{\text {step }}$ & $\begin{array}{l}\text { Modelled after von Mises distribution (equation } 7 \text { ) with concentration } \\
\text { parameter, } \kappa^{30} \text {. Can be subdivided into cue-detection, if applicable cue- } \\
\text { transfer, in-flight cue-maintenance and drift errors (equation } 6 \text {, Fig. 1). }\end{array}$ \\
\hline \multirow[t]{5}{*}{$\begin{array}{l}\text { Compass } \\
\text { courses }\end{array}$} & Geographic loxodrome & $\begin{array}{l}\text { Constant heading relative to perceived geographic axis (equation 14), } \\
\text { identifiable (within-step) by a time-compensated star or sun compass, or } \\
\text { by averaging polarized light cues at dawn and dusk. }\end{array}$ \\
\hline & Geomagnetic loxodrome & Constant heading relative to perceived geomagnetic axis (equation 15). \\
\hline & Magnetoclinic & $\begin{array}{l}\text { Stepwise geomagnetic heading based on maintaining a fixed transverse } \\
\text { projection of proximate inclination }{ }^{13} \text { (equation } 16,17 \text { in dipole). }\end{array}$ \\
\hline & Fixed sun compass & Constant heading vs. sunrise or sunset azimuth (equation 19). \\
\hline & $\begin{array}{l}\text { Time-compensated sun } \\
\text { compass (TCSC) }\end{array}$ & $\begin{array}{l}\text { As in fixed sun compass but offset due to longitudinal clock-shift relative } \\
\text { to internal clock }{ }^{14} \text {, affecting perceived sunset azimuth (equation 20-22). } \\
\text { We also quantify how the TCSC offset varies with a migrant's reference } \\
\text { (step) for sun azimuth rotation (equation 24), which could be local or } \\
\text { when clocks are reset during extended stopover (equation } 23 \text {, Figure 6). }\end{array}$ \\
\hline \multirow{5}{*}{$\begin{array}{l}\text { Performance } \\
\text {-related } \\
\text { factors (for } \\
\text { independent } \\
\text { steps on a } \\
\text { plane) }\end{array}$} & $\begin{array}{l}\text { Migratory performance, } \\
p_{\text {Arr }}\end{array}$ & $\begin{array}{l}\text { Probability of successfully reaching destination, i.e., arriving within the } \\
\text { goal area. }\end{array}$ \\
\hline & Goal area & Migratory destination, modelled by goal radius, $R_{\text {goal }}$ (radians). \\
\hline & Migration distance, $R_{\text {mig }}$ & Distance (radians) from initial step (e.g., natal site) to centre of goal area. \\
\hline & Goal-area breadth, $\beta$ & Goal-area radius divided by migration distance, $R_{\text {mig }}$. \\
\hline & $\begin{array}{l}\text { Length-adjusted goal- } \\
\text { area breadth, } \beta_{\text {adj }}\end{array}$ & $\begin{array}{l}\text { Goal-area breadth, } \beta \text {, multiplied by square root of minimum (error-free) } \\
\text { number of steps, } N_{0} \text {. Governs performance in the normal planar limit } \\
\text { (Fig. 1, equations } 11-13 \text { ) }\end{array}$ \\
\hline
\end{tabular}


If we unwrap a single flight-step, stepwise precision will itself depend on (initial) cue detection, cue maintenance (i.e., in-flight cue redetermination; Fig. 1b), and any cue transfer (Fig. 1c). For flight-steps based on a single cue (Fig. 1b), cue maintenance will reduce expected stepwise errors (Supplementary Fig. 1b-c, equation 8a) at the expense of stepwise flight distance ${ }^{31}$. However, for flight-steps involving cue transfer to a second compass (Fig. 1c), cue maintenance cannot make up for initial cue detection and transfer errors (Supplementary Fig. 1b, equation 8b). Therefore, within a single nocturnal flight-step, non-transferred geomagnetic or star-compass headings are relatively more precise compared with headings transferred to a second compass (assuming equivalent precision among compasses in cue detection and maintenance).

\section{Compass course formulations and sensitivity}

In the Methods, we formulated stepwise compass headings for each compass courses (Table 1, equations 14-17, 19, 22). For interpretability across global scales, we formulated magnetoclinic courses assuming a geomagnetic dipole model, in which magnetoclinic headings vary solely with geomagnetic latitude (equation 17). We further extended "classic" TCSC courses sensu Alerstam $^{14}$, to quantify how resetting of a migrant's inner clock and, additionally, possible use of proximate sun-azimuth rotation affect its "time-compensated" offset relative to any "clock-shift" caused by crossing longitudes equations (22-24).

The heuristics of TCSC migration and self-correction are illustrated in Fig. 2. Following errorfree headings, a migrant's subsequent heading will shift oppositely to its clock shift, creating an increasingly Southward trajectory (Northward in the Southern Hemisphere). Following an imprecise heading, the error-induced "time-compensation" offset (equation 21) will therefore naturally tend to counteract any (erroneous) difference in clock-shift. 


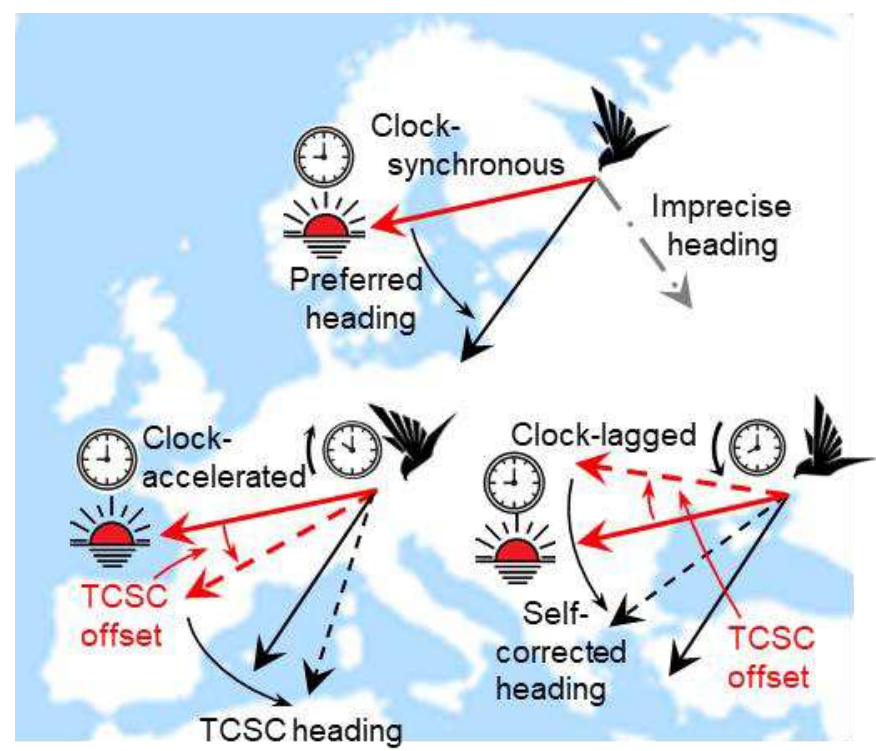

Fig. 2. Time-compensated sun compass (TCSC) headings and self-correction.

A TCSC migrant clock-synchronized to local conditions (above) maintains its preferred direction (solid black arrow) by adjusting its heading relative to the daily clockwise rotation in sun azimuth (here at sunset, solid red arrow). Following an error-free flight-step (lower left), the longitudinally (here, Westward) displaced migrant will be clock-shifted (here, clock-accelerated) relative to local time. This results in an "over-compensation" to proximate sun azimuth, i.e., counter-clockwise TCSC offset (dashed red arrow), hence more Southward (here, less Westward) heading (dashed black arrow). If the migrant's initial heading is imprecise (dot-dashed grey line), its stepwise longitudinal displacement will lead to a contrasting clock-shift (here, clock-lag). The now clock-lagged migrant (lower right) will "undercompensate" relative to proximate sun azimuth, resulting in a clockwise offset (dashed red arrow) and hence self-corrected heading (dashed black line). Between-step shifts in proximate sunset azimuth (not shown) become biologically relevant at multi-day and multi-step scales (Fig. 5). 
We quantified sensitivity to stepwise error algebraically as iterative (proportional) growth in errors of stepwise headings, revealing contrasting latitudinal and directional patterns, with large ranges in iterative growth in errors including partial self-correction (Fig. 3). Preferred geographic loxodrome headings (equation 14) will per definition not depend on previous headings, resulting in "zero" growth or correction in error as long as cue-detection errors are stepwise independent (Fig. 3a). This also holds for geomagnetic loxodrome headings in a dipole field (relative to geomagnetic axes, equation 15). Contrastingly, the latitude-dependence of magnetoclinic headings (equation 17) renders them stepwise inter-dependent, and leads to extremely high sensitivity for virtually any non-Southerly heading at both high and low latitudes (Fig. 3b, equation 25). Errors in fixed sun compass courses remain largely stepwise independent (close to "zero" growth), but will iteratively grow or self-correct at high latitudes, depending on whether East or West oriented, and before or after the fall equinox (equation 26, Fig. 3c-d). Sensitivity in TCSC headings is similarly East-West antisymmetric about the equinox (Fig. 3e-f), but their self-correcting nature (Fig. 2) renders them relatively insensitive, with 5- 25\% stepwise selfcorrection over a broad range of directions (equation 27), into which headings (blue arrows) moreover tend to "converge". While the degree of stepwise TCSC correction remains small away from polar latitudes (as shown in Fig. 2, roughly to scale), subsequent steps will also partially self-correct for any discrepancy in longitude as long as inner clocks are not reset. 
Fig. 3.
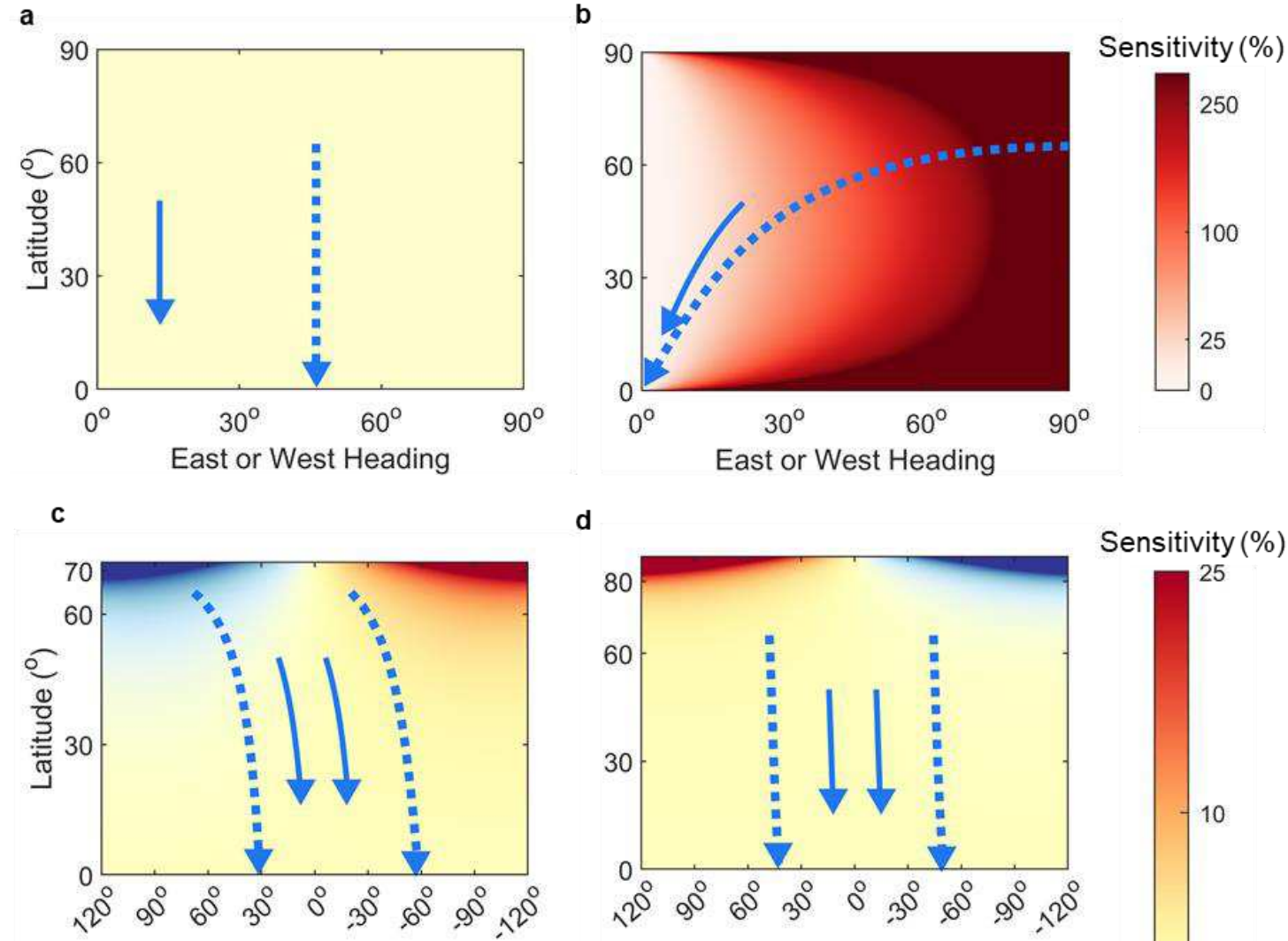

d
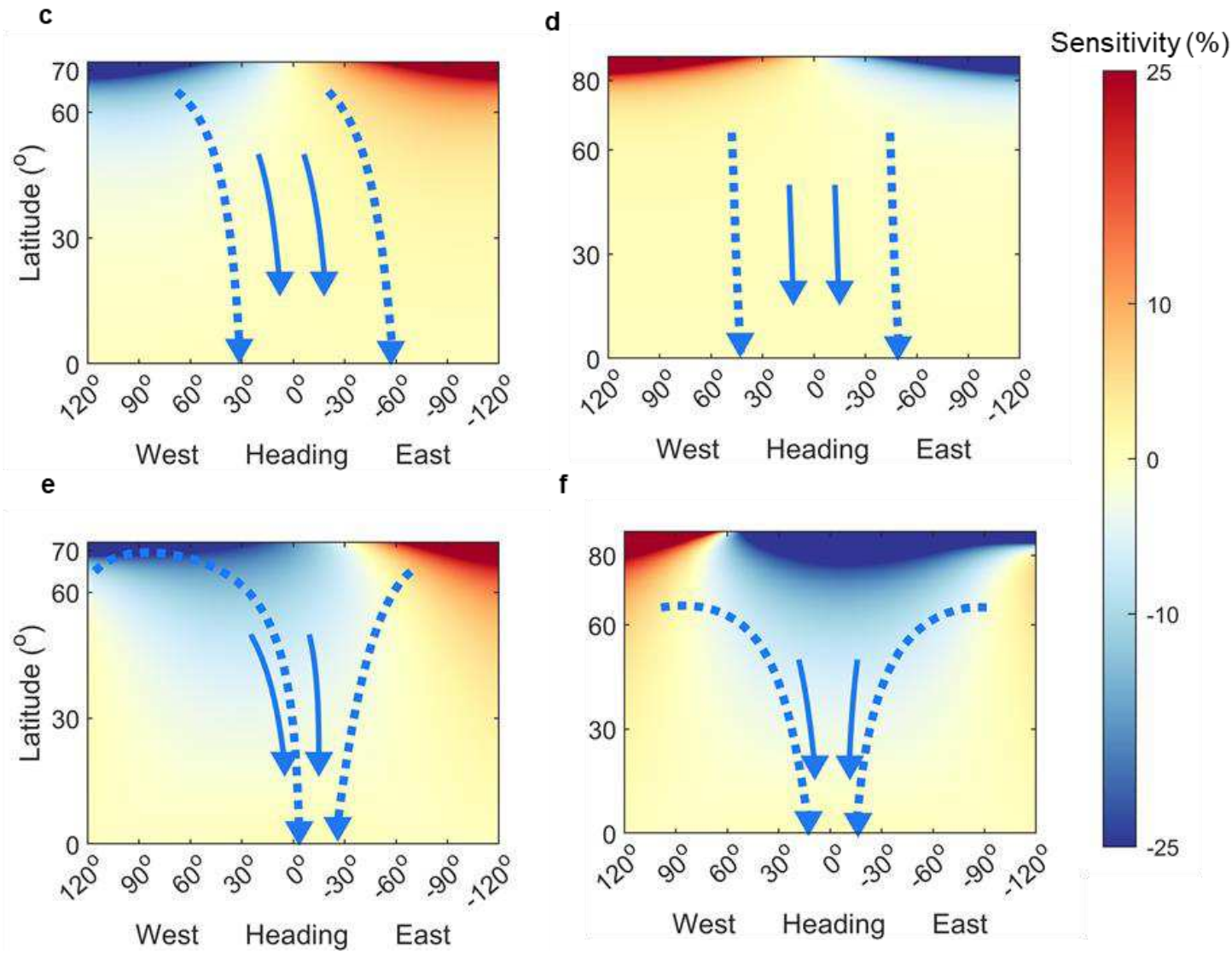


\section{Fig. 3. Stepwise sensitivity varies strongly with heading and among compass courses.}

Stepwise sensitivity, i.e., iterative growth of small errors in heading (\%, with colour scales on right), as a function of current heading (clockwise from South) and latitude (geomagnetic South and geomagnetic latitude for geomagnetic courses), for (a) constant-heading geographic loxodromes, or equivalently geomagnetic loxodromes in a geomagnetic dipole Earth, (b) magnetoclinic courses in a geomagnetic dipole, (c) fixed sun compass courses on August $1^{\text {st }}$ and (d) October $1^{\text {st }}$, and (e) time-compensated sun compass (TCSC) courses on August $1^{\text {st }}$ and (f) October $1^{\text {st }}$. For positive (yellow to red coloured) values of sensitivity, errors in stepwise heading will grow iteratively, whereas for negative (white to blue coloured) values, headings are self-correcting. Blue arrows depict error-free headings for travel from (solid lines) $50^{\circ} \mathrm{N}-15^{\circ} \mathrm{N}$ across $10^{\circ}$ in longitude, and (dashed lines) $65^{\circ} \mathrm{N}-0^{\circ} \mathrm{N}$ across $90^{\circ}$ in longitude. For all simulations, stepwise movements were $360 \mathrm{~km}$. In (c-f), regions without sunset or sunrise (poleward of $\sim 72^{\circ}$ on August $1^{\text {st }}$ and $\sim 87^{\circ}$ on October $1^{\text {st }}$, respectively) are not depicted. 


\section{Simulation of migration routes}

For each species and compass course, route-optimized trajectories, i.e., with headings maximizing performance (probability of successful arrival), are illustrated in Fig. 4 and Supplementary Figure 3, with key model parameters for all nine species listed in Table 2. With total effective stepwise errors of $20^{\circ}$, among-species performance generally increased with length-adjusted goal-breadth (Figs. 4b, Supplementary Fig. 3a), which governs performance in the planar normal limit (equation 11). TCSC courses always performed best, and magnetoclinic courses much worse than all other compass courses, with geomagnetic loxodromes also less consistent. However, when incorporating biologically-relevant within-step and betweenindividual variability (Figure. 4b-f, Supplementary Fig. 3c-g), TCSC courses only outperformed loxodrome courses along the non-transferred Monarch Butterfly (Danaus plexippus) route (Fig. 4b) and long-distance night-migratory Willow Warbler (Phylloscopus trochilus) and Graycheeked Thrush (Catharus minimus) routes (Fig. 4e-f). For all three species, TCSC courses most closely matched the known routes simulated. For the ca. 14,000 km Willow Warbler route and also near West-East migration of Common Rosefinch (Carpodacus erythrinus) (Fig. 4c), magnetoclinic courses were virtually intractable. Cue-transferred courses are presented for a nocturnal star compass, but transfers to a geomagnetic in-flight compass performed overall very similarly (Supplementary Fig. 4).

Diversity in compass-cue favourability for Marsh Warbler (Acrocephalus palustris) migration over a range of (component-wise) errors is illustrated in Fig. 5, including greater drift tolerance among TCSC courses (Fig. 5i-j) and a slight advantage of geomagnetic over geographic loxodromes, particularly when the latter are based on polarized light, e.g., when the star compass is unavailable on departures (Fig. 5a-b, e-f). 
Table 2. Model parameters of the species compass course simulations. Species and routes, ordered by migration distance, used in model simulations to assess compass course performance. Routes and migration pace were based on tracking and other studies, including initial departure dates \pm standard deviation (and maximum arrival date), great-circle (followed by loxodrome) distances and headings, flight (ground) speed, travel (migration) speeds, and migration schedule, the latter modelled as a (fixed) sequence of consecutive flight steps followed by an extended stopover (mean \pm standard deviation). Length-adjusted goal breadth, $\beta_{a d j}$ (equation 13), governs performance in the normal planar limit (equation 11, Fig. 3a). All migrants except the Monarch Butterfly are principally night-migratory.

\begin{tabular}{|c|c|c|c|c|c|c|c|c|c|c|c|c|c|}
\hline Species (reference) & Route & $\begin{array}{l}\text { Departure } \\
\text { date }\end{array}$ & $\begin{array}{l}\text { Distance } \\
(\mathrm{km})\end{array}$ & $\begin{array}{l}\Delta \text { Lat } \\
\Delta \text { Lon }\end{array}$ & $\begin{array}{l}\text { Initial } \\
\text { heading } \\
\left({ }^{\circ}\right)\end{array}$ & $\begin{array}{l}\text { Goal } \\
\text { radius } \\
(\mathbf{k m})\end{array}$ & $\begin{array}{l}\text { Migratory } \\
\text { breadth }\end{array}$ & $\begin{array}{l}\text { Flight } \\
\text { speed } \\
(\mathrm{m} / \mathrm{s})\end{array}$ & $\begin{array}{l}\text { Stepwise } \\
\text { distance } \\
(\mathrm{km})\end{array}$ & $\begin{array}{l}\text { Consecutive } \\
\text { flight steps, } \\
\text { stopover } \\
\text { duration (d) }\end{array}$ & $\begin{array}{l}\text { Travel } \\
\text { speed } \\
(\mathbf{k m} / \mathbf{d})\end{array}$ & $\begin{array}{l}\text { Minimum } \\
\text { (maximum) } \\
\text { flight steps }\end{array}$ & $\begin{array}{l}\text { Length- } \\
\text { adjusted } \\
\text { goal } \\
\text { breadth } \\
\end{array}$ \\
\hline $\begin{array}{l}\text { Monarch Butterfly, }{ }^{12,32} \\
\text { Danaus plexippus }\end{array}$ & $\begin{array}{l}\text { Quebec- } \\
\text { Mexico }\end{array}$ & $\begin{array}{l}\text { Aug } 15 \pm 14 \\
(\sim \operatorname{Dec} 13)\end{array}$ & $\begin{array}{l}3290 / \\
3300\end{array}$ & $\begin{array}{l}28^{\circ} / \\
30^{\circ}\end{array}$ & $\begin{array}{l}219 \\
(213)\end{array}$ & 100 & 0.03 & 3.0 & 85 & $5,3 \pm 1$ & 55 & $36.9(77)$ & 0.18 \\
\hline $\begin{array}{l}\text { Ring Ouzel }{ }^{33}, \text { Turdus } \\
\text { torquatus }\end{array}$ & $\begin{array}{l}\text { Scotland - } \\
\text { N Africa }\end{array}$ & $\begin{array}{l}\text { Aug } 31 \pm 7 \\
(\sim \operatorname{Nov} 29)\end{array}$ & $\begin{array}{l}2610 / \\
2610\end{array}$ & $\begin{array}{l}24^{\circ} / \\
1^{\circ}\end{array}$ & $\begin{array}{l}181 \\
(179)\end{array}$ & 250 & 0.10 & 11.5 & 330 & $10,15 \pm 5$ & 130 & $7.1(42)$ & 0.26 \\
\hline $\begin{array}{l}\text { Common Rosefinch, }{ }^{34} \\
\text { Carpodacus erythrinus }\end{array}$ & $\begin{array}{l}\text { Bulgaria - } \\
\text { NW India }\end{array}$ & $\begin{array}{l}\text { Aug } 7 \pm 7 \\
(\sim \text { Nov } 29)\end{array}$ & $\begin{array}{l}5110 / \\
5170\end{array}$ & $\begin{array}{l}18^{\circ} / \\
52^{\circ}\end{array}$ & $\begin{array}{c}96 \\
(123)\end{array}$ & 400 & 0.08 & 12.5 & 360 & $5,5 \pm 2$ & 400 & $13.1(44)$ & 0.28 \\
\hline $\begin{array}{l}\text { Marsh Warbler, }{ }^{35} \\
\text { Acrocephalus palustris }\end{array}$ & $\begin{array}{l}\text { Finland - } \\
\text { Kenya }\end{array}$ & $\begin{array}{l}\text { Sep } 1 \pm 7 \\
(\sim \operatorname{Jan} 1)\end{array}$ & $\begin{array}{l}6720 / \\
6730\end{array}$ & $\begin{array}{l}60^{\circ} / \\
10^{\circ}\end{array}$ & $\begin{array}{l}168 \\
(173)\end{array}$ & 500 & 0.07 & 11.5 & 330 & $5,5 \pm 2$ & 165 & $18.8(48)$ & 0.32 \\
\hline $\begin{array}{l}\text { Kirtland's Warbler, }{ }^{36} \\
\text { Setophaga kirtlandii }\end{array}$ & $\begin{array}{l}\text { Michigan - } \\
\text { Bahamas }\end{array}$ & $\begin{array}{l}\text { Oct } 6 \pm 7 \\
(\sim \operatorname{Dec} 5)\end{array}$ & $\begin{array}{l}2370 / \\
2370\end{array}$ & $\begin{array}{l}21^{\circ} / \\
7^{\circ}\end{array}$ & $\begin{array}{l}157 \\
(160)\end{array}$ & 300 & 0.13 & 10 & 290 & $5,5 \pm 2$ & 145 & $7.2(33)$ & 0.34 \\
\hline $\begin{array}{l}\text { Nathusius Bat, }{ }^{37} \\
\text { Pipistrellus nathusii }\end{array}$ & $\begin{array}{l}\text { Latvia - } \\
\text { Spain }\end{array}$ & $\begin{array}{l}\text { Aug } 15 \pm 14 \\
(\sim \text { Nov } 13)\end{array}$ & $\begin{array}{l}2040 / \\
2050\end{array}$ & $\begin{array}{l}13^{\circ} / \\
20^{\circ}\end{array}$ & $\begin{array}{l}233 \\
(224)\end{array}$ & 300 & 0.15 & 7.5 & 160 & $3,5 \pm 2$ & 60 & $10.8(36)$ & 0.48 \\
\hline $\begin{array}{l}\text { Willow Warbler, }{ }^{38} \\
\text { Phylloscopus trochilus } \\
\text { yakutensis }\end{array}$ & $\begin{array}{l}\text { Siberia - } \\
\text { Zambia }\end{array}$ & $\begin{array}{l}\text { Sep } 1 \pm 7 \\
(\sim \operatorname{Jan} 1)\end{array}$ & $\begin{array}{l}13,200 / \\
14,600\end{array}$ & $\begin{array}{l}80^{\circ} / \\
138^{\circ}\end{array}$ & $\begin{array}{l}311 \\
(233)\end{array}$ & 1000 & 0.08 & 10.5 & 300 & $5,2 \pm 2$ & 215 & $40.2(87)$ & 0.48 \\
\hline $\begin{array}{l}\text { Gray-cheeked Thrush, }{ }^{39} \\
\text { Catharus minimus }\end{array}$ & $\begin{array}{l}\text { Yukon - } \\
\text { Columbia }\end{array}$ & $\begin{array}{l}\text { Sep } 10 \pm 7 \\
(\sim \operatorname{Jan} 7)\end{array}$ & $\begin{array}{l}9080 / \\
9300\end{array}$ & $\begin{array}{l}65^{\circ} / \\
70^{\circ}\end{array}$ & $\begin{array}{l}108 \\
(141)\end{array}$ & 1000 & 0.11 & 11.5 & 330 & $5,5 \pm 2$ & 165 & $24.4(63)$ & 0.54 \\
\hline $\begin{array}{l}\text { Eurasian Hoopoe, }{ }^{40} \\
\text { Uрира ерорs }\end{array}$ & $\begin{array}{l}\text { Switzerland } \\
- \text { W Africa }\end{array}$ & $\begin{array}{l}\text { Aug } 10 \pm 7 \\
(\sim \text { Oct } 9)\end{array}$ & $\begin{array}{l}3370 / \\
3380\end{array}$ & $\begin{array}{l}29^{\circ} / \\
10^{\circ}\end{array}$ & $\begin{array}{l}204 \\
(200)\end{array}$ & 800 & 0.24 & 12.0 & 345 & $5,5 \pm 2$ & 170 & $7.4(33)$ & 0.65 \\
\hline
\end{tabular}



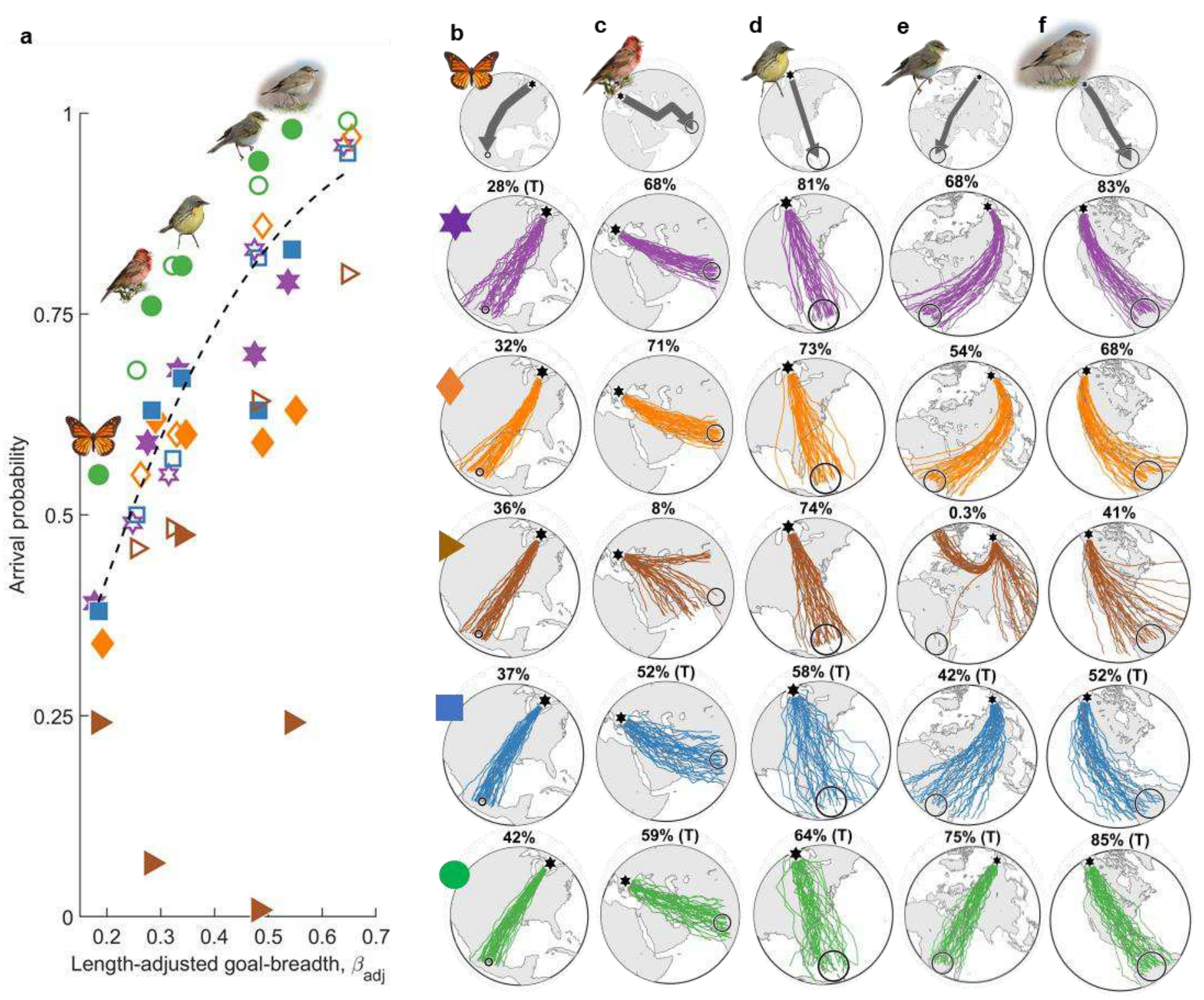

Fig. 4. 


\section{Fig 4. Diverse compass course performance among species and migration routes. (a)}

Compass-route performance, assuming $20^{\circ}$ total stepwise equivalent error, vs. length-adjusted goal

breadth, which governs expected performance (dashed line) in the normal planar limit (equations 11-13), for 9 species (Table 2), with filled symbols representing (left-right) Monarch Butterfly, Common open symbols representing the other species (depicted in Supplementary Fig. 3). Purple hexagons represent geographic loxodromes, orange diamonds geomagnetic loxodromes, brown triangles magnetoclinic courses, blue squares fixed sun compass courses and green circles time-compensated sun compass (TCSC) courses. (b-f) Randomly-sampled route-optimal trajectories for each compass course, with matching colours and symbol in (b), for the above-named species (with the others depicted in Supplemental Fig. 3), assuming biologically-relevant variability (with $15^{\circ}$ cue detection, maintenance, drift and, where applicable, transfer errors, and $2.5^{\circ}$ inter-individual variability in inherited headings). Known species migratory courses, from natal grounds (black hexagons) to natural goal areas (open circles), depicted by grey arrows (great circles appear as straight lines in the stereographic projection). Performance (\%) and, where applicable, also cue-transferred courses (“T”) are depicted above each panel. All TCSC courses are cue-transferred and all geographic loxodrome courses are non-transferred star compass courses, except for Monarch butterfly migration, for which it is the other way around. For the biologically-relevant scenario depicted, cue-transfers nearly doubled effective stepwise errors compared with non-transferred courses ( $28^{\circ}$ vs. $\sim 16^{\circ}$ degrees, equation 10$)$. Photos of (b-d) from Wikimedia, and (e) by HS. 

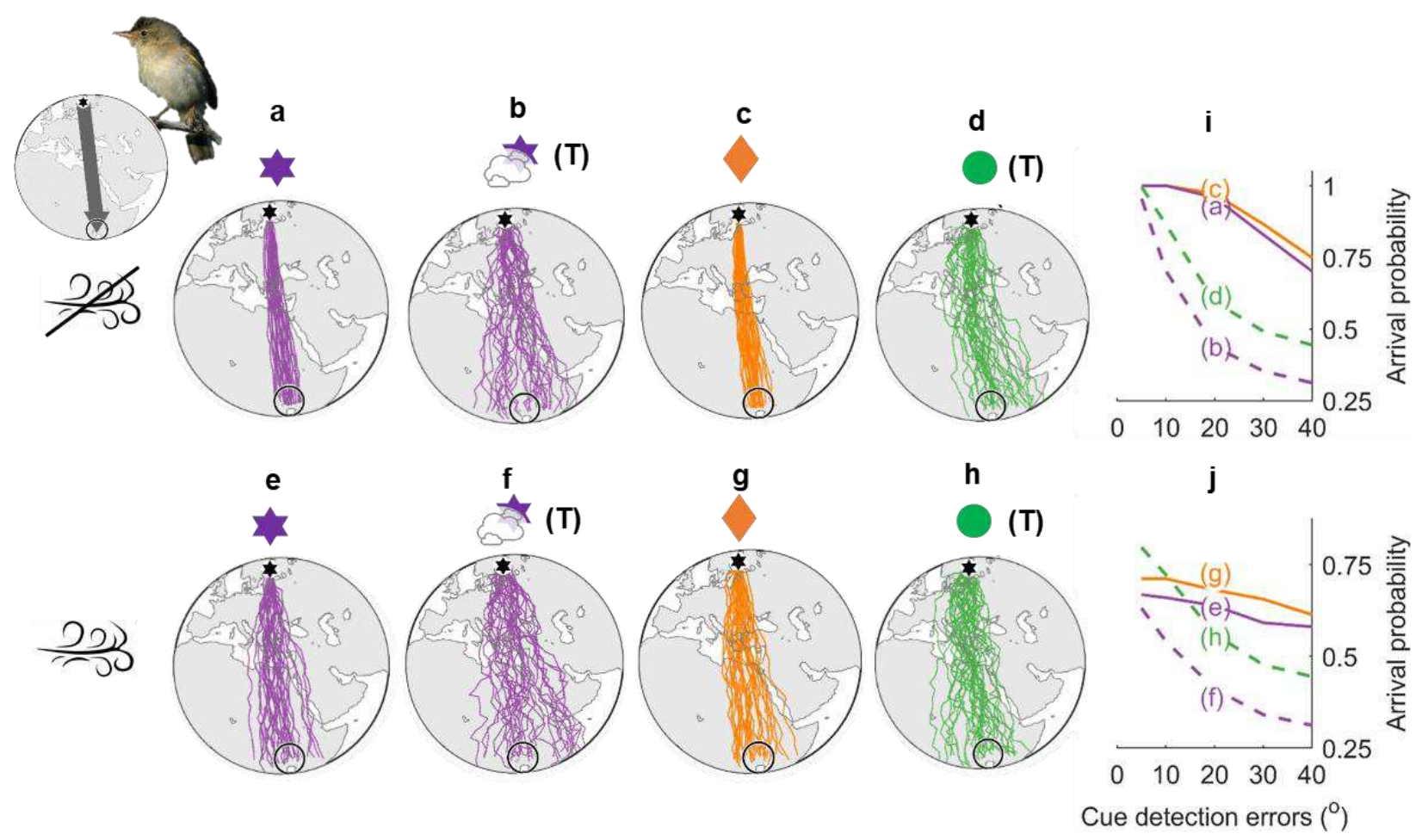

Fig. 5. Effect of cue detection, cue transfer and drift on Marsh Warbler migration

Trajectories and arrival probabilities of simulated Marsh Warbler (Acrocephalus palustris, photo Wikimedia) migration (grey arrow in inset and Table 2, but with fixed migratory schedules) between breeding grounds in Finland (black hexagons) and wintering grounds in East Africa (open circles, radius $500 \mathrm{~km}$ ), assuming (a-d) $20^{\circ}$ effective errors in within-step cue detection, cue transfer, and hourly cue maintenance and (e-h) additionally, $20^{\circ}$ hourly in-flight drift. Symbols and trajectory-colours are as in Fig. 3: (a, e) geographic loxodrome courses using a non-transferred star compass, (b, f) geographic loxodrome using a cue-transferred polarized light to star compass (e.g., if cloud cover prevents the star compass on departure), (c, g) non-transferred geomagnetic loxodrome course, and (d, h) timecompensated sun compass courses (using either polarized light or sun azimuth) transferred to a star compass. The effects of varying the magnitude of within-step cue errors on the arrival probability is shown for the cases (i) without drift and (j) with $20^{\circ}$ hourly drift, colour-coded by primary compass type, with solid lines for non-transferred (loxodrome) courses and dashed lines for courses transferred to a star compass (with depicted scenarios a-h annotated). 
The relative performance among compass courses over a range of effective errors was further supported by the global compass-course simulations of generic migrants in a geomagnetic dipole (Supplementary Fig. 5). The global simulations additionally revealed systematically narrower longitudinal ranges in feasible routes for magnetoclinic courses, and overall lower maximal stepwise errors for feasible migration at high-latitudes. Simulations of biologically-relevant error scenarios (Supplementary Fig. 6) further revealed narrower longitudinal ranges for high-latitude fixed compass courses, and heterogeneity in the performance gain of both sun-compass courses relative to non-transferred loxodrome courses, with TCSC courses losing their self-correcting advantage (cf. Fig. 4 a) with effective within-step errors exceeding $\sim 30^{\circ}$ Supplementary Fig. 6 ).

10 The effects of inner-clock resetting and time-compensation across continental scales are illustrated in Fig. 6 for simulated Gray-cheeked Thrush migration (with known routes in inset $^{39,41}$ ). "Classic" TCSC trajectories (Fig. 6a) resemble both great circles and known routes but rely on stepwise (nightly) headings always being adjusted according to sun-azimuth rotation rates as experienced on departure from the natal grounds. Contrastingly, when adjusting nightly

15 headings to proximate sun-azimuth rotation (Fig. 6b), trajectories deviate strongly from great circles, unless (Fig. 6c) inner-clocks are reset and headings retained during extended stopovers. Finally, trajectories vary more strongly and contrastingly with departure date when migrants inherit sun compass headings (Fig. 6d) as opposed to geographic headings (Fig. 6a-c). 


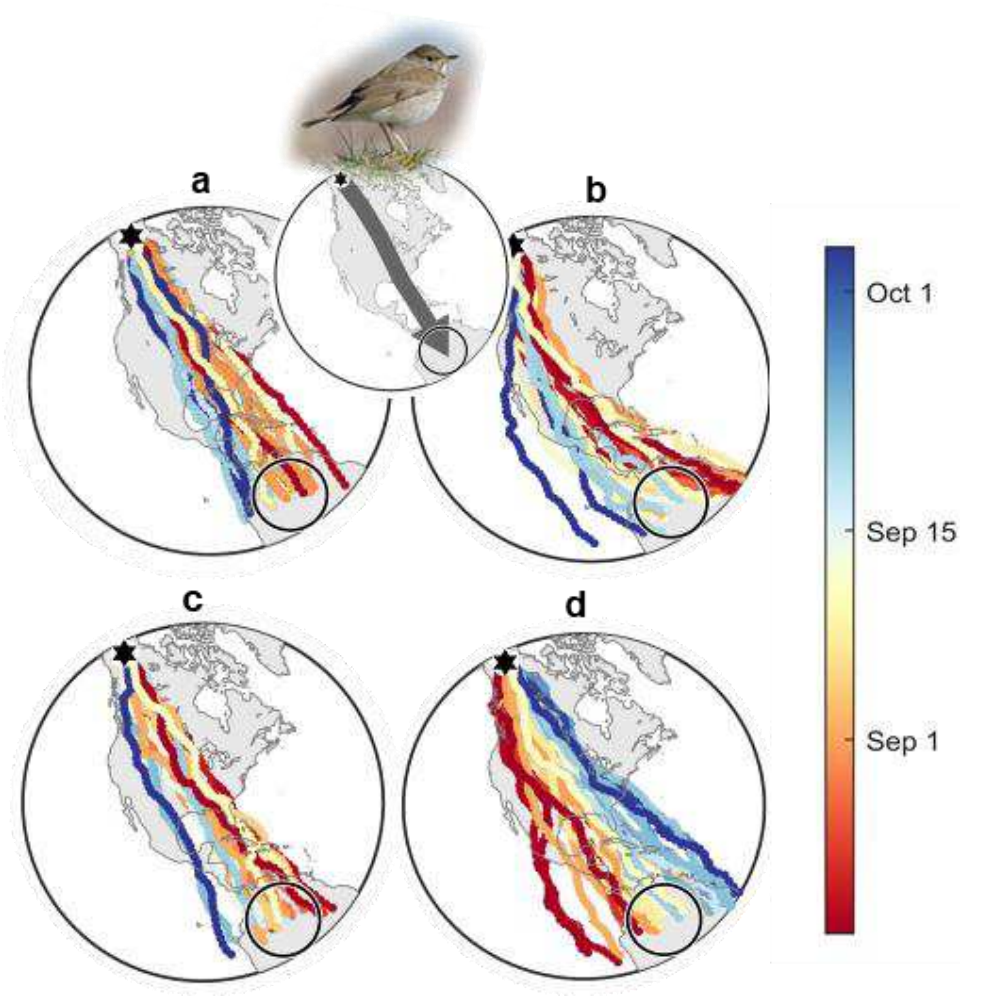

Fig. 6. Extended sun-compass formulation illustrates time-compensation, latitudinal and inner-clock effects. Route and schedule optimized time-compensated sun compass (TCSC) trajectories (randomly selected from 10,000 modelled individuals) with $20^{\circ}$ effective stepwise standard errors, modelled after Gray-cheeked Thrush (Catharus minimus) migration (grey arrow in inset) from Yukon, Canada (black hexagons) to Columbia (open circles), with trajectories colour-coded for initial departure date (mean 10 Sept. \pm 14 days, i.e., double the range compared with in Table 2 and Fig. 4). Great circles appear as straight lines in the stereographic projection. (a) With uninterrupted ("non-stop") nightly flights and, sensu ${ }^{14}$, adjustments in stepwise heading gauged according to (hourly) sun-azimuth rotation retained from the natal grounds (equation 20). (b) As in (a), but with stepwise heading-adjustments based on proximate (local) rather than natal-site rates of sun-azimuth rotation (equation 24a). (c) Based on local sun-azimuth rotation as in (b), but with sequences of 5 flights interspersed by 5 -day stopovers, during which geographic headings are retained and inner-clocks reset (equation 24b); (d) As in (c), but where optimized headings are inherited as offsets to proximate sun-azimuth rather than as geographic headings, hence varying with departure date. Photo by Alix D’Entrement. 


\section{Factors governing compass-course performance}

We used regression and model selection to diagnose and fit the extent to which spherical geometry and compass-course sensitivity effects modulate effective stepwise error (Fig. 1b), including how performance increases with increasing number of steps, $N$ (introducing a generalized exponent $g$, comparing equations 11 to 34). We also accounted for seasonal constraints on performance via a population-specific maximum number of steps (Table 2, equation 28). We focused on diagnosing relative performance of the overall best-performing loxodrome and TCSC courses, with differences between geographic and geomagnetic loxodromes indicating non-dipole (geomagnetic declination) effects. We expected the relative performance gain of cue-transferred TCSC courses among night-migratory species to increase with three "performance gain" factors: a route-specific multiplicative spherical geometry factor ( $G \geq 1$, equation 31 ), the minimum number of flight steps, $N_{0}$, and stepwise flight distance. For interpretability, we present results for simulations using total stepwise error without drift or between-individual variability in initial (inherited) headings.

Fig. 7a-i depicts performance of geographic loxodromes (solid purple hexagon), geomagnetic (solid orange hexagons) and TCSC courses (solid green circles) as a function of total stepwise error among species (shown in increasing order of the product of the three performance gain factors), and also AICc model-selected regression fits for geographic loxodromes (open hexagons) and TCSC courses (open circles) incorporating the above factors $\left(R_{a d j}^{2} \geq 0.97\right.$, see Supplementary Tables 1-2). Considering equivalent stepwise errors, TCSC courses once again always outperformed both loxodromes, with geomagnetic loxodromes performing less consistently than geographic loxodromes. Among biologically-relevant scenarios $\left(5^{\circ}-25^{\circ}\right.$ effective component-wise error), performance gain with TCSC compared with loxodromes 
varied as predicted relative to $N_{0}$ and the spherical geometry factor (Fig. 7j), with the highlatitude Gray-cheeked Thrush and Willow Warbler and daytime migrant Monarch Butterfly (Figs. 7e, h-i) once again predicted to benefit most from TCSC courses. The trade-off between self-correction and cue transfer in the favourability of TCSC courses was reflected in the modelselected regression coefficients (Supplementary Table 2), with baseline performance of TCSC courses predicted to increase faster with number of steps compared with loxodrome courses, but also "decaying" nearly twice as rapidly with increasing stepwise effective error. Erroraugmentation due to the spherical geometry factor was also three times larger along geomagnetic loxodrome courses compared with geographic loxodrome or TCSC courses, reflecting heightened sensitivity to crossing bands of geomagnetic longitude (e.g., ${ }^{42}$ ).

Fig 7.
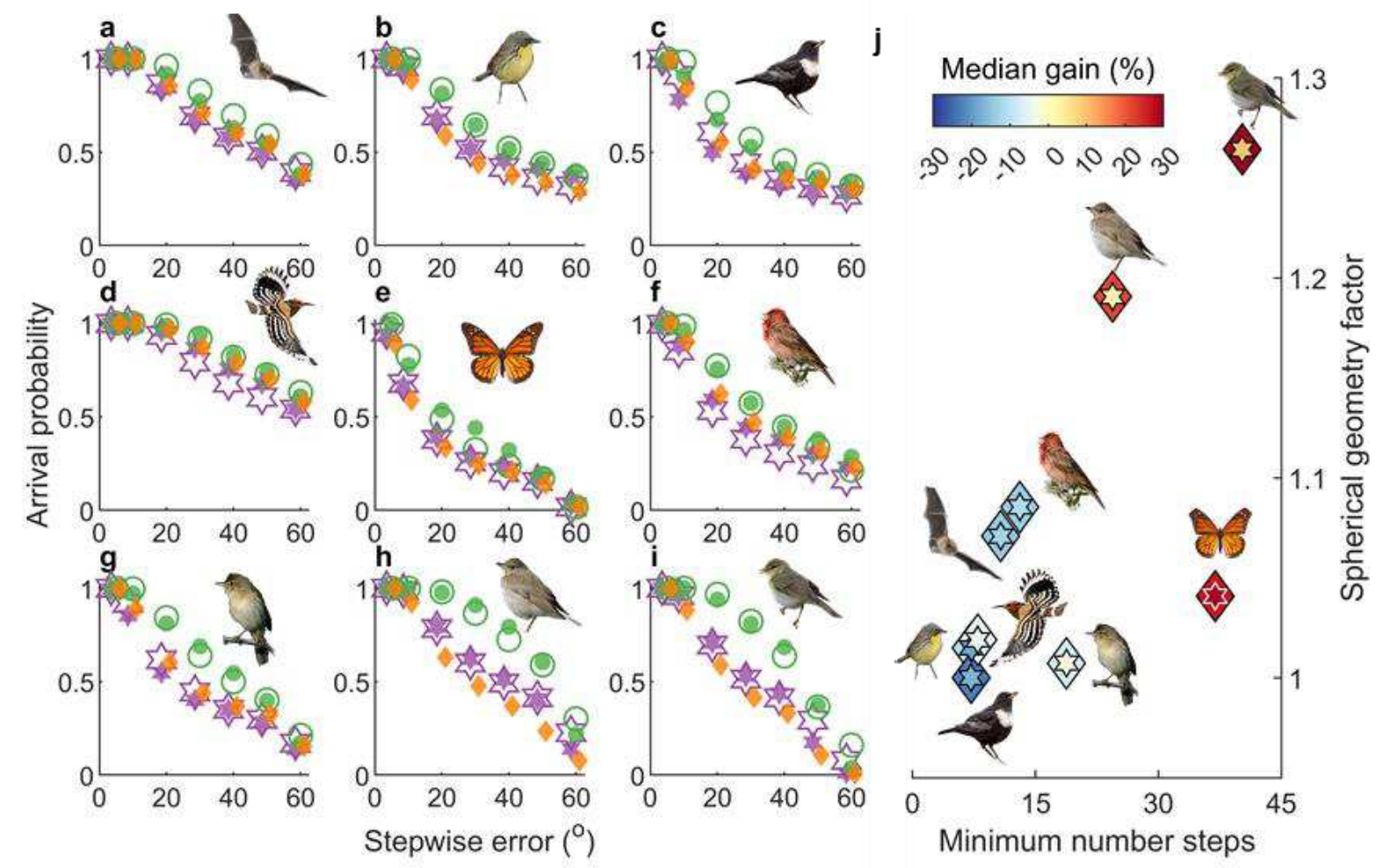


\section{Fig 7. Relative compass-course performance and cue favourability predicted by distance}

and spherical geometry factors. (a-i) Route-optimized performance (arrival probability) vs. total stepwise standard error per modelled species (Table 2) for geographic loxodrome (purple hexagons, starcompass except for Monarch Butterfly, (e)), geomagnetic loxodrome (orange diamonds, right-adjusted for visibility), and TCSC courses (green circles). Open symbols depict model-selected regression-estimated performance for geographic loxodromes (hexagons) and TCSC courses (circles), including parameters for factors governing convergence in mean heading with number of steps, geographic (longitudinal) effects and (for TCSC courses) stepwise flight distance. (j) Median percentile gains in performance of TCSC courses vs. geographic loxodromes (colour-coded inner hexagons) and geomagnetic loxodromes (colourcoded outer diamonds) for biologically-relevant variability $\left(5^{\circ}-25^{\circ}\right.$ within-step effective error components and between-individual variability), as a function of required steps and the spherical geometry factor (equation 32), which increases with longitudes crossed and initial latitude (Supplementary Fig. 7). Photographs of (a) by Roger Jones, (c, g) from Wikicommons and (d) by Gordon Langsbury. 


\section{Discussion}

Our extended formulation of compass courses has facilitated a first global assessment of factors governing robustness of inaugural migration routes, and provides an explanation for enhanced

5 performance by naïve migrants. We propose that unassisted inaugural migratory performance is mediated by the required goal breadth and minimum number of flight steps (Figs. 1, 4), and relative favourability among compass-courses by a readily-derived spherical geometry factor (equation 32, Supplementary Fig. 7), the minimum number of steps, and stepwise flight distance (Fig. 7). While cue transfers compound effective stepwise errors to reduce overall performance, unbiased in-flight cue maintenance will improve performance with little penalty in stepwise distance (Supplementary Fig. 1c-d). Our simulation results based on hourly cue-maintenance indicate, consistently with radar measurements of nocturnally migrating birds, that magnitudes of expected cue detection, transfer and within-flight errors should remain below about $30^{\circ}$ (circular lengths $<0.85)^{31}$. Motion and cue related effects presumably limit the effectiveness of higherfrequency cue maintenance.

Augmentation of effective errors through spherical geometry effects (Figs. 4, 7, Supplementary Figs. 5-6) has been largely ignored in animal migration and navigation studies ${ }^{16,18,24,25}$, and presents a further challenge with high-latitude migration next to better recognized limitations in cue availability and precision ${ }^{42,43}$. Unlike inexperienced migrants without a map sense ${ }^{2}$, early European nautical explorers overcame analogous challenges by developing transverse Mercator map projections ${ }^{23}$. Naïve migrants at high latitudes could mitigate errors by flying for shorter numbers of hours, e.g., during early-autumn, which would automatically reduce longitudinal displacements. 
The contrasting stepwise sensitivity of the compass courses (Fig. 3) have strong implications regarding their adaptive value to migratory populations. We propose that magnetoclinic migration routes are highly unlikely to have evolved given their general high sensitivity and poor performance along strongly direction-changing routes (for which they were envisaged ${ }^{13}$ ), with loxodrome or sun compass courses performing equivalently well or better along nearly Southward routes (Fig. 4, Supplementary Figs. 3, 5-6). Contrastingly, as an emergent "many slightly-corrected wrongs" phenomenon, TCSC courses are ubiquitously more robust compared with fixed sun-compass courses, even outweighing the penalty of cue-transfers and more closely matching known routes in comparison to non-transferred loxodrome courses for the longestdistance and most high-latitude night-migratory populations tested. For most other nightmigratory routes, geomagnetic loxodrome courses and star-compass courses performed best in biological scenarios (Fig. 4, Supplementary Fig. 3), at least assuming equivalent cue precision and availability, with slight advantages for geomagnetic courses among simulated migrants in Europe and for celestial courses in North America. When stars are not visible on departure, starcompass courses transferred from more ubiquitously available polarized light cues performed less well (Figs. 4-5), and moreover rely on averaging of cues at dusk and dawn (often from different locations) in order to diagnose geographic South ${ }^{8,10}$. A further advantage of nocturnal TCSC courses is in similarly being achievable using polarized light cues but only at either dusk or dawn, avoiding the need to average cues. Our results support that continental-scale TCSC courses can be robust to variable schedules and to updating of hourly rates of time-compensation en route (Fig. 6, equation 26), at least if inner clocks are updated and headings maintained during stopovers. Indeed, flight directions of high-latitude bird migrants ${ }^{14,44}$ and some migratory insects $^{2}$ most closely resemble TCSC headings, and stepwise-calibration using twilight cues may 
be most prevalent among longer-distance migrants ${ }^{7,8}$. An important caveat to TCSC courses in pre-breeding (spring) migrations is that self-correction will not work for poleward movement, at least without integration with additional cues.

The finding that TCSC courses are self-correcting provides a novel explanation for naïve migrants overcoming otherwise prohibitive errors ${ }^{19}$, and observed route-corrections following displacement. Previous studies ${ }^{45}$ proposed naïve migrant self-correction relies on a timecompensating star compass, now generally regarded as not supported ${ }^{9,29}$. For the self-correction in TCSC courses based on sun azimuth described here, subsequent headings following displacement would be offset by equation (21), based on the displaced migrant's reference latitude (equation 24). Experimental evidence of self-correction following displacement by naïve migrants is inconsistent, and has also often been confounded by polar or equatorial cue effects $^{42,46}$, and probably by inner-clock updates ${ }^{9,47}$. For the perhaps clearest and most convincing case for naïve self-correction, by GPS-tagged Eurasian Cuckoos (Cuculus canorus) following a $28^{\circ}$ longitude displacement at $55^{\circ} \mathrm{N}^{20}$, the estimated shift in headings compared with non-displaced "control" individuals $\left(21^{\circ}\right)$ is intriguingly close to as predicted $\left(23^{\circ}\right)$ using equation (21).

Our results support observed diversity in migratory compass-cue hierarchy ${ }^{6-8}$, consistent with cue-conflict experiments, with celestial cues dominating among the most extreme routes and daytime migrants, and geomagnetic-calibrated orientation among most migrants in Europe ${ }^{7,8}$. Naturally, cue favourability is also contingent upon appropriate biological cue mechanisms ${ }^{2,6}$, and further modulated by relative cue precision and availability (particularly in polar regions or when crossing the equator ${ }^{6,11,18}$ ), as well as topography, habitat quality and weather factors ${ }^{2,48}$. For example, analysis of light-level geolocation data of Common Rosefinches migrating along 
the modelled route (Fig. 4c) revealed largely wind-driven movement with a detour around the Iranian Desert ${ }^{34}$, and it is not clear whether naïve Nathusius Bats display innate migratory directions $^{49}$. Our models can be readily extended to consider geographical and meteorological factors, as well as regarding the extent to which spatial variability in inherited headings ${ }^{50}$ can maintain routes given long-term spatiotemporal variability in the Earth's geomagnetic field ${ }^{27,51}$ or, for sun compass courses, seasonal migration schedules ${ }^{32,52}$. More generally from a movement ecology perspective, our study highlights that care must be taken when assessing movement without accounting for cue precision, and that, even in the age of big data and tracking ${ }^{53}$, models of simple responses in simplified environments can still reveal novel emergent effects with potentially profound life-history implications. 


\section{Methods}

\section{Stepwise directed movement on a sphere}

Terms defining stepwise movement, precision and geophysical orientation cues are listed in

Table 1. Since seasonal migration nearly ubiquitously proceeds from higher to lower latitudes, it is convenient to define headings clockwise from geographic South (counter-clockwise from geographic North for migration initiated in the Southern Hemisphere). Given stepwise headings on a sphere, $\alpha_{i}$, with $i=0, \ldots, N-1$, stepwise latitudes, $\emptyset_{i+1}$ and longitudes, $\lambda_{i+1}$, can be calculated using the Haversine equation ${ }^{54}$, which can be approximated by stepwise planar movement:

$$
\begin{aligned}
& \emptyset_{i+1}=\emptyset_{i}-R_{\text {step }} \cos \alpha_{i}, \\
& \lambda_{i+1}=\lambda_{i}-R_{\text {step }} \sin \alpha_{i} / \cos \emptyset_{i}
\end{aligned}
$$

Here, the stepwise distance $R_{\text {step }}=3.6 V_{a} \cdot n_{H} / R_{\text {Earth }}$ (in radians), relative to the mean Earth radius $R_{\text {Earth }}(\mathrm{km})$, depends on the migrant's flight speed, $V_{a}(\mathrm{~m} / \mathrm{s})$, and stepwise flight hours, $n_{H}$. For improved accuracy and to accommodate within-step effects, we updated headings and locations hourly, $h=1, \ldots, n_{H}$

$$
\begin{gathered}
\emptyset_{i, h}=\emptyset_{i, h-1}-\frac{R_{s t e p}}{n_{H}} \cos \alpha_{i, h-1}, \\
\lambda_{i, h}=\lambda_{i, h-1}-\frac{R_{\text {step }}}{n_{H}} \sin \alpha_{i, h-1} / \cos \emptyset_{i, h-1},
\end{gathered}
$$

where $\alpha_{i, h}$ are hourly in-flight headings relative to geographic South. In the absence of drift effects (see below), migrants were assumed to retain their preferred (i.e., expected) headings from stepwise departures ${ }^{17,48}$, either by accounting for (hourly) sun or star rotation, or else relative to a geomagnetic axis ${ }^{6,7}$. Accordingly, for a geomagnetic in-flight compass, expected 
headings, $\bar{\alpha}_{i, h}$, are modulated by changes in the magnetic declination, $\delta_{m, i, h}$, i.e., the clockwise difference between geographic and geomagnetic South ${ }^{6}$ :

$$
\bar{\alpha}_{i, h}=\left\{\begin{aligned}
\bar{\alpha}_{i, 0}, & \text { celestial compass } \\
\bar{\alpha}_{i, 0}+\delta_{m, i, h}-\delta_{m, i, 0}, & \text { geomagnetic compass }
\end{aligned}\right.
$$

Between-step and within-step errors

5 Stepwise errors were modelled in two ways: 1 ) considering (total) stepwise error, $\varepsilon_{i, h}=\varepsilon_{i}^{\text {step }}$, relative to a fixed within-step heading; 2) considering biologically relevant scenarios, including cue-detection error, $\varepsilon_{i}^{d e t}$, where applicable, stepwise cue-transfer errors, $\varepsilon_{i}^{\text {trans }}$, in-flight (default, hourly) cue maintenance errors, $\varepsilon_{i, h}^{m n t}$, and drift errors, $\varepsilon_{i, h}^{d r f t}$, i.e.,

$$
\varepsilon_{i, h}=\left\{\begin{aligned}
\varepsilon_{i}^{d e t}+\varepsilon_{i, h}^{m n t}+\varepsilon_{i, h}^{d r f t}, & \text { no cue tranfer } \\
\varepsilon_{i}^{d e t}+\varepsilon_{i}^{\text {trans }}+\varepsilon_{i, h}^{m n t}+\varepsilon_{i, h}^{d r f t}, & \text { with cue tranfer }
\end{aligned}\right.
$$

Drift errors represent a proxy for wind ${ }^{17,55}$, topography ${ }^{56}$ or variability in compass cues ${ }^{51}$, without explicitly considering wind strength effects. Estimated cue precision of the avian compasses lie between $0.5^{\circ}$ and $10^{\circ} 7,43,57$, in-flight errors equivalent to about $20^{\circ}-30^{\circ} 31$, and stepwise precision including drift effects typically between $10^{\circ}$ and $50^{\circ} 16,17,35$. Finally, estimated variability in between-individual preferred headings is typically less than $10^{\circ} 17,50$.

Stepwise and in-flight errors were simulated using a von Mises distribution, defined by an angular "concentration" parameter, $\kappa$, analogous to the reciprocal of variance in headings:

$$
p(\alpha \mid \bar{\alpha}, \kappa)=\frac{1}{2 \pi I_{0}(\kappa)} e^{\kappa \cos (\alpha-\bar{\alpha})}
$$

where $I_{j}$ is the modified Bessel function of the first kind and order $j^{30}$. For sufficiently small concentrations, $\kappa$, von Mises samples are similar to normally sampled variables with "effective 
standard error", $\sigma=1 / \sqrt{\kappa}^{30}$. However, unlike sums of normal variables, circular random errors do not sum in a scale-free way, or necessarily even follow the same distribution as their components $^{30,58}$. Therefore, to assess compass courses, it is convenient to first consider the case of independent stepwise normal movement on a plane ${ }^{16,24}$, and then extend this to account for $5 \quad$ circular error $^{5,25,59}$, spherical geometry effects ${ }^{23}$ and, for non-loxodrome courses, interdependence of headings.

To estimate effective stepwise errors of single flight steps, we can apply the normal relations for the sum of two variables $\left(\sigma_{A+B}=\sqrt{\sigma_{A}^{2}+\sigma_{B}^{2}}\right)$ to any pre-flight cue transfer, and the average of $m$ variables of uniform standard deviation $\left(\sigma_{m}=\sigma / \sqrt{m}\right)$ to cue detection followed by $M$ cue maintenance events ${ }^{30}$ :

$$
\sigma_{\text {step }} \approx\left\{\begin{array}{cl}
\sigma / \sqrt{M+1}, & \text { no cue transfer } \\
\sigma \sqrt{2+M^{-1}}, & \text { with cue transfer }
\end{array}\right.
$$

Equation (8) indicates that cue-maintenance reduces expected stepwise errors for non-transferred flight (Equation 8a, Fig. 1b), but not so for flight with cue transfers (equation 8b, Fig. 1c). The accuracy of equation (8a-b) for circular stepwise errors with effective error components (colour scale on right) below $\sim 30^{\circ}$, and the trade-off between increased accuracy and decreased stepwise distance with increasing number of steps, is illustrated in Supplementary Figure 1.

We can analogously estimate effective standard error after $N$ steps for a single individual,

$$
\sigma_{N} \cong \sigma_{\text {step }} / \sqrt{N}
$$


or within a migratory population, considering both within-individual effective error following the expected number of steps, $\widehat{N}$, and between-individual variability in preferred (inherited) headings, $\sigma_{\text {ind }}$ :

$$
\sigma_{p o p} \cong \sqrt{\sigma_{\text {ind }}^{2}+\sigma_{\text {step }}^{2} / \widehat{N}}
$$

5 Equation 10 reflects the importance of relatively low between-individual variability ${ }^{24,35}$.

\section{Migratory performance on a plane}

Performance (arrival probability) of independent stepwise planar movement to a (circular) goal area of radius $R_{\text {goal }}$ will approximate a cumulative normal distribution (erf function), based on the breadth of successful angles and overall effective error, which is modulated by the expected number of steps. For long-distance migration, successful angles follow the goal-area breadth (Fig. 1, Table 1$)$, since $\beta=R_{\text {goal }} / R_{\text {mig }} \cong \tan ^{-1}\left(R_{\text {goal }} / R_{\text {mig }}\right)$. Assuming uniform population headings and applying equation (9) and the Central Limit Theorem for large numbers of steps, a first planar approximation to sufficiently directionally accurate migration is

$$
\hat{p}_{\beta, \widehat{N}} \approx p\left(\left|\left(\frac{1}{\widehat{N}} \sum_{i=1}^{\widehat{N}} \alpha_{i}\right)-\bar{\alpha}\right| \leq \beta\right) \approx \operatorname{erf}\left(\frac{\beta}{\sqrt{2} \sigma_{\text {step }} / \sqrt{\widehat{N}}}\right)
$$

where $\widehat{N}=N_{0} \cdot I_{1}\left(\kappa_{\text {step }}\right) / I_{0}\left(\kappa_{\text {step }}\right)$ is the expected number of steps, $\kappa_{\text {step }} \cong \sigma_{\text {step }}{ }^{-25,30,59}$, and

$$
N_{0}=\left(R_{\text {mig }}-R_{\text {goal }}\right) / R_{\text {step }}
$$

is the minimum (error-free) number of steps to reach the closest edge of the goal area. From equation (11) we see that within the planar and normal limit, i.e., high stepwise concentrations, $\kappa_{\text {step }}$, performance roughly follows the "length-adjusted goal breadth",

$$
\beta_{a d j}=\beta \sqrt{N_{0}}=\beta \sqrt{R_{\text {mig }} / R_{\text {step }}} .
$$




\section{Formulation of compass course headings}

Since sun compass headings vary with date, to ensure temporally consistent flight directions from the initial (natal) site with sun compass courses, we assumed that preferred headings were imprinted from inherited geographic or geomagnetic headings ${ }^{2,6,7}$.

Expected stepwise geographic headings remain unchanged en route, i.e.,

$$
\bar{\alpha}_{i}=\bar{\alpha}_{0}
$$

Expected stepwise geomagnetic headings remain unchanged relative to proximate geomagnetic South, i.e., are offset by stepwise declination en route

$$
\bar{\alpha}_{i}=\bar{\alpha}_{0}+\delta_{m, i}
$$

Magnetoclinic compass headings

As described and illustrated in detail in ${ }^{13}$, the magnetoclinic compass was hypothesized to explain the prevalence of "curved" migratory bird routes, i.e., for which local geographic headings gradually but significantly shift en route. Magnetoclinic compass courses involve a migrant adjusting its current heading to maintain a constant transverse component, $\gamma^{\prime}$, of the experienced inclination angle (see Supplementary Fig. 2), so that error-free stepwise headings are

$$
\bar{\alpha}_{i}=\sin ^{-1}\left(\frac{\tan \gamma_{i}}{\tan \gamma^{\prime}}\right)=\sin ^{-1}\left(\frac{\tan \gamma_{i} \sin \bar{\alpha}_{0}}{\tan \gamma_{0}}\right) .
$$

To assess magnetoclinic headings globally, we assumed a geomagnetic dipole field based on magnetic latitude, $\emptyset_{m}$, and which explains $90 \%$ of the Earth's magnetic variation ${ }^{60}$. Since the 
horizontal and vertical fields in a magnetic dipole are $B_{h}=B \cos \emptyset_{m}$ and $B_{z} \cong 2 B \sin \emptyset_{m}$, magnetic inclination, $\gamma$, is purely a function of magnetic latitude, $\gamma\left(\emptyset_{m}\right)=\tan ^{-1}\left(B_{z} / B_{h}\right)=$ $\tan ^{-1}\left(2 \tan \emptyset_{m}\right)$. Therefore, the projected transverse component becomes

$$
\gamma^{\prime}=\tan ^{-1}\left(\frac{\tan \gamma_{0}}{\sin \bar{\alpha}_{0}}\right)=\tan ^{-1}\left(\frac{2 \tan \emptyset_{m, 0}}{\sin \bar{\alpha}_{0}}\right)
$$

and, in order to maintain a constant projection, $\gamma^{\prime}$, stepwise magnetoclinic headings in a geomagnetic dipole field follow

$$
\bar{\alpha}_{i}=\sin ^{-1}\left(\frac{2 \tan \emptyset_{m, i}}{\tan \gamma^{\prime}}\right)=\sin ^{-1}\left(\frac{\sin \bar{\alpha}_{0}}{\tan \emptyset_{m, 0}} \tan \emptyset_{m, i}\right)
$$

\section{Sunrise and sunset azimuth}

In Supplementary Information 1, we derive a simple formula for sunset azimuth, $\theta_{s}$, which aids interpretation of sun compass courses and computational efficiency when simulating large numbers of modelled individuals,

$$
\theta_{s}=\left\{\begin{array}{c}
\cos ^{-1}\left(-\sin \delta_{s} / \cos \emptyset\right) \\
180^{\circ}(24 \text {-hour light }) \\
0^{\circ}(24 \text {-hour dark })
\end{array}\right.
$$

where only the positive (i.e., West of South) solution is taken, and the solar declination, $\delta_{s}$, varies cyclically between $-23^{\circ}$ and $23^{\circ}$ through the year (Supplementary Information 1 . Sun compass courses are also achievable based on other times, in particular at sunrise (sunrise azimuth is the exact negative of sunset azimuth relative to geographic South) and using polarized light cues at either sunrise and sunset, during which the maximum band of polarized light is perpendicular to sun azimuth, i.e., $\theta_{\text {pol }}=\theta_{s}-\pi / 2$. 


\section{Fixed sun compass headings}

Fixed sun-compass headings represent a uniform (clockwise) offset, $\bar{\alpha}_{s}$ relative to the spatiotemporally-shifting sun azimuth, $\theta_{s, i}$,

$$
\bar{\alpha}_{i}=\bar{\alpha}_{s}+\theta_{s, i}
$$

where, to ensure consistent initial flight directions at the initial (natal) site, the preferred heading $\bar{\alpha}_{s}=\bar{\alpha}_{0}-\theta_{s, 0}$ is presumed to be imprinted using an innate geographic or geomagnetic heading.

\section{Time-compensated sun compass (TCSC)}

Even outside the realm of migration, many insects ${ }^{58,61}$, and birds $s^{28,57}$ are known to use a timecompensated sun compass to maintain preferred directions locally, by accounting for the daily rotation in sun azimuth. In a pioneering work addressing migration, Alerstam and Pettersson ${ }^{14}$ made the link between the "clock-shift" induced by crossing bands of longitude (meridians), $\Delta \mathrm{h}$ $=24 \cdot \Delta \lambda / 2 \pi$, and its effect on a migrant adjusting its heading to the (hourly) rotation of the sun's azimuth,

$$
\frac{\partial \theta_{s}}{\partial h} \cong \frac{2 \pi \sin \emptyset}{24}
$$

resulting in an offset to their interpretation of sun azimuth, and therefore to their "timecompensated" offset on departure at sunset:

$$
\Delta \alpha \cong \Delta \lambda \sin \emptyset
$$

Equation (21) results in near-great-circle trajectories for small ranges in latitude, $\emptyset$ and until inner clocks are reset (also resetting $\Delta \lambda$ ). As proposed by Alerstam ${ }^{14}$, TCSC courses can be extended if migrants both reset their clocks and retain migratory directions during extended stopover. However, all simulations have further assumed that the migrant also adjusts its heading 
based on sun-azimuth rotation rates (equation 20) from the original (or most recent stopover) site and latitude, which may not be the case (migrants may also respond to proximate rates of sun azimuth, without resetting their inner clocks). We therefore extended the formulation to track inner clock and time-compensating "reference steps" independently:

$$
\bar{\alpha}_{i}=\left\{\begin{array}{rl}
\bar{\alpha}_{c_{r e f, i}}+\left(\theta_{s, i}-\theta_{s, c_{r e f, i}}\right)+\left(\lambda_{i}-\lambda_{c_{r e f, i}}\right) \sin \emptyset_{s_{r e f, i}}, & i \neq c_{r e f, i} \\
\alpha_{i-1}, & i=c_{r e f, i}
\end{array},\right.
$$

with the index $c_{r e f, i}$ specifying the current clock reset site, where the previous heading is also imprinted,

$$
\bar{\alpha}_{c_{r e f, i}}=\left\{\begin{aligned}
\bar{\alpha}_{0}, & i=0 \\
\bar{\alpha}_{c_{r e f, i-1},} & i>0, i \neq c_{r e f, i} \\
\alpha_{i-1}, & i>0, i=c_{r e f, i}
\end{aligned}\right.
$$

and $s_{r e f, i}$ specifying the reference step for "time-compensated" adjustments to sun azimuth (equation 20):

$$
s_{r e f, i}=\left\{\begin{aligned}
c_{r e f, i}, & \text { classic TCSC } \\
i, & \text { proximately gauged TCSC }
\end{aligned}\right.
$$

\section{Sensitivity of compass course headings}

Sensitivity was assessed by the marginal change in expected heading from previous headings, $\partial \bar{\alpha}_{i} / \partial \alpha_{i-1}$; when this is positive, small errors in headings, and therefore migratory trajectories, have "zero" sensitivity. 
For magnetoclinic compass courses in a dipole field, stepwise sensitivity can be calculated by differentiating equation (17) with respect to previous headings:

$$
\frac{d \bar{\alpha}_{i}}{d \alpha_{i-1}}=\frac{\sin \bar{\alpha}_{0}}{\tan \emptyset_{m, 0}} \cdot \frac{1}{\cos \bar{\alpha}_{i} \cos ^{2} \emptyset_{m, i}} \frac{\partial \emptyset_{m, i}}{\partial \alpha_{i-1}}=\frac{R_{s t e p} \sin \alpha_{i-1} \sin \bar{\alpha}_{0}}{\cos \bar{\alpha}_{i} \cos ^{2} \emptyset_{m, i} \tan \emptyset_{m, 0}}
$$

All three terms in the denominator indicate, as illustrated in Fig. 3b, that magnetoclinic courses become unstably sensitive at both high and low latitudes, and any heading with a significantly East-West component.

Sensitivity of fixed sun compass headings is non-zero due to sun azimuth dependence on location (equation 18):

$$
\begin{gathered}
\frac{d \bar{\alpha}_{i}}{d \alpha_{i-1}}=\frac{\sin \delta_{s, i}}{\sin \theta_{s, i}} \cdot \frac{\sin \emptyset_{i}}{\cos ^{2} \emptyset_{i}} \frac{\partial \emptyset_{i}}{\partial \alpha_{i-1}}=\frac{\sin \delta_{s, i}}{\sin \theta_{s, i}} \cdot \frac{R_{s t e p} \sin \emptyset_{i} \sin \alpha_{i-1}}{\cos ^{2} \emptyset_{i}} \\
=R_{\text {step }} \cdot \sin \alpha_{i-1} \frac{\tan \emptyset_{i}}{\tan \theta_{s, i}}
\end{gathered}
$$

The sine factor on the right-hand side in equation (26) causes the sign of $\partial \bar{\alpha}_{i} / \partial \alpha_{i-1}$ to be opposite for East to West or West to East headings, and $\tan \theta_{s}$ also changes sign at the fall equinox (due to solar declination changing sign). The azimuth term in the denominator indicates heightened sensitivity closer to the summer or winter equinox and at high latitudes ${ }^{9}$, and, conversely, reduced sensitivity (robustness) close to the spring or autumnal equinox (since $\left.\tan \theta_{s, 0} \rightarrow \pm \infty\right)$. This seasonal and directional asymmetry is illustrated in Figs. $3 \mathrm{c}$ and 3e.

TCSC courses (equation 22) involve up to three sensitivity terms:

$$
\frac{d \bar{\alpha}_{i}}{d \alpha_{i-1}}=R_{s t e p} \cdot \sin \alpha_{i-1} \frac{\tan \emptyset_{i}}{\tan \theta_{s, i}}+\frac{d \lambda_{i}}{d \alpha_{i-1}} \sin \emptyset_{c_{r e f}, i}+\left(\lambda_{i}-\lambda_{c_{r e f}, i}\right) \frac{d \sin \emptyset_{s_{r e f}, i}}{d \alpha_{i-1}}
$$


$=\left\{\begin{array}{r}R_{s t e p} \cdot\left[\sin \alpha_{i-1} \frac{\tan \emptyset_{i}}{\tan \theta_{s, i}}-\frac{\cos \alpha_{i-1} \sin \emptyset_{s_{r e f}, i}}{\cos \emptyset_{i-1}}\right], \text { classic (27a) } \\ R_{\text {step }}\left[\sin \alpha_{i-1} \frac{\tan \emptyset_{i}}{\tan \theta_{s, i}}-\frac{\cos \alpha_{i-1} \sin \emptyset_{s_{r e f}, i}}{\cos \emptyset_{i-1}}+\left(\lambda_{i}-\lambda_{c_{r e f}, i}\right) \sin \alpha_{i-1} \cos \emptyset_{i}\right], \text { proximate (27b). }\end{array}\right.$

The first square-bracketed terms in equations (27a-b) are identical to with the fixed sun compass (equation 26), reflecting seasonal and latitudinal dependence in sun-azimuth. For headings with a Southward component $\left(\alpha_{0}<90^{\circ}\right)$, the second bracketed terms are always negative, i.e., sensitivity-reducing, resulting in a broad range in latitude and headings with self-correcting headings (Fig. 3c-f). The third bracketed terms in equation (27b) with proximate TCSC is also negative, and in fact increasingly so until clocks are reset, bur remains small in magnitude compared to the second term.

\section{Spatiotemporal orientation and movement model}

To assess the feasibility and robustness of each compass course to spatiotemporal effects on a global scale, we simulated inaugural migration based on equations (3-27) for both a generic migrant across all feasible longitudinal ranges (hereafter, global simulations) and for 9 contrasting airborne species (hereafter species simulations) chosen for diversity among taxa, latitude and longitude ranges and goal-area breadths (Table 2). For consistency with our focus of a single (inherited) compass heading, we avoided migratory routes with extensive open-ocean flights or sudden direction shifts. In several cases (e.g., Common Rosefinch, Eurasian Hoopoe Ring Ouzel and Nathusius Bat), modelled non-breeding ranges may represent subsets rather than ubiquitous migratory destinations among the breeding population. Migrants flew for a specific number $\left(n_{f l}\right)$ of uninterrupted daily or nightly steps before making extended stopovers for $n_{f l}$ days to "refuel"24,62. Note that these stopover schedules do not preclude extensive pre- 
migratory fuelling, often found among long-distance migratory birds ${ }^{38,41,63}$. Simulated generic migrants departed Sept $15^{\text {th }} \pm 5$ days (mean \pm standard deviation, rounded to the nearest day), flying for 3 consecutive nights at flight (ground) speeds of $12.5 \mathrm{~m} / \mathrm{s}$, followed by $5 \pm 2$ days stopover. For all species and routes, given stepwise $\left(R_{\text {step }}\right)$ and migratory $\left(R_{\text {mig }}\right)$ flight

5 distances, and the ratio of stopover to flight days $\left(1+n_{s t o p} / n_{f l}\right)$, the minimum $\left(N_{0}\right.$, equation 12) and maximum $\left(N_{\max }=T_{\max } /\left(1+n_{\text {stop }} / n_{f l}\right)\right.$ number of steps can be determined, where $T_{\max }$ is the population-specific maximum migration duration in days (Table 2).

For the global compass course simulations, we simulated migration in all cardinal directions (in $1^{\circ}$ increments) for medium-distance migration at mid-latitudes $\left(45^{\circ}-25^{\circ} \mathrm{N}\right)$ and long-distancemigration beginning at high latitudes $\left(65^{\circ} \mathrm{N}-0^{\circ}\right)$, assuming a goal radius of $500 \mathrm{~km}$ and migration on a geomagnetic dipole Earth (i.e., ignoring declination effects). For the species simulations, we incorporated spatiotemporally dynamic geomagnetic data (MATLAB 2020b package igrf) ${ }^{27}$, assuming a default season, fall 2000. Optimal headings maximizing arrival probabilities were determined using the MATLAB nonlinear solver fminbnd, for initial loxodrome and magnetoclinic headings between $-90^{\circ}$ and $90^{\circ}$ (clockwise SE to SW), and initial sun compass headings between $-145^{\circ}$ and $145^{\circ}$, which can begin with Northward headings ${ }^{11,18}$. Modelled migration was terminated once migrants passed $1000 \mathrm{~km}$ South of the goal area or maximum number of steps, $N_{\max }$.

We assessed robustness of the global and species simulations in two ways: 1) for effective total stepwise standard errors of $0^{\circ}-60^{\circ}$, i.e., ignoring schedule-related or further sources of variability, and 2) for biologically-relevant scenarios incorporating within-step cue detection, transfer and maintenance errors (assuming equivalent magnitudes in standard error), variability in migratory departure and stopovers (Table 2), as well as effective standard errors of $2.5^{\circ}$ in 
inherited (between-individual) headings ${ }^{50}$ and $15^{\circ}$ in hourly in-flight drift, presumed to be autocorrelated ${ }^{51,55}$ with hourly (coefficient 0.75 ) and also between flight-steps (coefficient 0.25 ), but not following extended stopovers.

\section{Accounting for seasonal constraints, spherical-geometry and self-correction effects}

\section{$5 \quad$ Seasonal migration constraints}

In assessing performance, we also accounted for seasonal migration constraints via a populationspecific maximum number of steps, $N_{\max }$ (Table 2; this became significant for the longestdistance simulations with large magnitudes equivalent errors). Using the Central Limit Theorem and known properties of sums of $\operatorname{cosines} C_{j}(N)=\frac{1}{N} \sum_{i=1}^{N} \cos \left(j \cdot \alpha_{i}\right)^{30,59}$, this is

$$
p_{\emptyset, N_{\text {max }}} \cong p\left(R_{\text {step }} \sum_{i=1}^{N_{\max }} \cos \left(\alpha_{i}\right) \geq R_{\text {mig }} \cos \bar{\alpha}\right) \cong \frac{1}{2}\left[1-\operatorname{erf}\left(\left(\frac{N_{0}}{N_{\max }}-\frac{I_{1}(\kappa)}{I_{0}(\kappa)}\right) \cdot \frac{\cos \bar{\alpha}}{\sigma_{C} \sqrt{2}}\right)\right],
$$

where $E\left[\left(C_{j} \mid \bar{\alpha}\right)\right]=\cos (j \bar{\alpha}) \cdot I_{j}\left(\kappa_{\text {step }}\right) / I_{0}\left(\kappa_{\text {step }}\right)$, and

$$
\sigma_{C}^{2}=\operatorname{Var}\left(\left(C_{1} \mid \bar{\alpha}\right)\right)=\frac{1}{2} \cdot\left(1+E\left[C_{2}\right]-2 E\left[C_{1}\right]^{2}\right) .
$$

\section{Spherical-geometric modulation of longitude errors}

On the sphere, stepwise longitude (equation 2) naturally contains a secant factor, i.e., cosine of latitude in the denominator, reflecting the convergence of meridians (bands of longitude) with increasing latitude. This secant factor causes the sensitivity of stepwise longitude to stepwise headings to increase with latitude:

$$
\frac{d \lambda_{i}}{d \alpha_{i-1}} \cong-\frac{R_{\text {step }} \cos \alpha_{i-1}}{\cos \emptyset_{i-1}}
$$


meaning that orientation errors at higher latitudes will exert a greater influence on overall longitudinal error, for any compass course. Due to this secant factor, the effective route-mean longitudinal error will scale approximately as in a Mercator projection ${ }^{23}$ :

$$
L=\frac{1}{\left(\emptyset_{0}-\emptyset_{A}\right)} \int_{\emptyset_{A}}^{\emptyset_{0}} \frac{d \emptyset}{\cos \varnothing}=\frac{1}{\left(\emptyset_{0}-\emptyset_{A}\right)} \ln \left(\tan \left(\frac{\emptyset_{0}+\emptyset_{A}}{2}\right)+\frac{\pi}{4}\right)
$$

where $\emptyset_{0}$ and $\emptyset_{A}$ are the initial (natal) and arrival latitude, respectively. To assess total error, the multiplicative factor $L$ will be modulated by the (mean) orientation en route:

$$
G=\sqrt{(\mathrm{L} \sin \bar{\alpha})^{2}+\cos ^{2} \bar{\alpha}}
$$

the scaling factor therefore being largest for purely Eastward or Westward headings $(G=L \geq 1)$ and nonexistent for North-South headings ( $G=1$, reflecting no longitude bands being crossed).

We further modified the effective goal-area breadth by a fixed factor to account for a (geographically) circular goal area on the sphere, i.e., effectively modulating the longitudinal component of the goal-area breadth at the arrival latitude, $\emptyset_{A}$ :

$$
\beta_{A}=\beta \sqrt{\sin ^{2} \bar{\alpha}+\left(\cos \bar{\alpha} / \cos \emptyset_{A}\right)^{2}}
$$

\section{Error sensitivity and error-correction effects}

To accommodate compass-course-specific sensitivity (iterative augmentation or self-correction in stepwise errors), we generalized the "normal" inverse-square-root relation between performance and number of steps (equations $11-12$ ), from $1 / \widehat{N}^{0.5}$, to $1 / \widehat{N}^{\eta}$, with

$$
\eta\left(\sigma_{\text {step }} \mid s, b\right)=(0.5+b) e^{-s \sigma_{s t e p}{ }^{2}}
$$


where $b<0$ reflects iterative augmentation of stepwise errors and $b>0$ self-correction, and $s$ represents an exponential damping factor, consistent with the limiting circular-uniform case (as $\kappa \rightarrow 0$, i.e., $\sigma_{\text {step }} \rightarrow \infty$ ), where no convergence of heading is expected with increasing step number (given modelled migration was terminated South of the goal area). \\ Assessing performance using regression and model selection}

For each compass course, based on route-optimized simulations among all 9 species, we fitted performance as the product of sufficiently timely migration (equation 27) and sufficiently accurate migration (equation 11), with the latter updated to account for the "non-normal" effects (equations 30-32), i.e., $p_{A r r}=p_{\emptyset, N_{\text {max }}} p_{\beta, \widehat{N}}$. Accordingly, we used MATLAB routine fitnlm based on the route-optimized species simulations and, to fit all combinations of up to four parameters for each compass course, and selected among models with parameter combinations using AICc, the Akaike information criterion corrected for small sample size ${ }^{64}$. Specifically, we accounted for

i) a compass-route specific fitted exponent, $g$, to the spherical geometry factor (equation 30), i.e., $G^{g}$, reflecting how sensitivity or self-correction in stepwise errors further augments or reduces this factor,

ii) a baseline offset, $b_{0}$, to $\eta=0.5$, as in equation (33),

iii) a fitted exponential damping factor $s$ with respect to stepwise error (equation 33), (iv) for TCSC courses, a fitted modulation $\rho$, quantifying the extent to which selfcorrection increases with increased stepwise distance $R_{\text {step }}$, i.e., $b=b_{0} R_{\text {step }}^{\prime}{ }^{\rho}$ in equation (33), where $R_{\text {step }}^{\prime}$ is the stepwise distance scaled by its median value among species.

To summarize, we generalized the formulation for sufficiently accurate migration as 


$$
p_{\beta, \widehat{N}} \cong \operatorname{erf}\left(\frac{\beta_{A}}{G^{g} \sqrt{2\left(\sigma_{\text {ind }}^{2}+\sigma_{\text {step }} / \widehat{N}^{n}\right)}}\right) \text {, }
$$

with $\eta\left(\sigma_{\text {step }} \mid s, b\right)=\left(0.5+b_{0}{R_{\text {step }}^{\prime}}^{\rho}\right) e^{-s \sigma_{\text {step }}{ }^{2}}$. Null values for the spherical geometry parameter were set to $g=1$, and for the parameters governing convergence of route-mean headings $b_{0}=0, s=0$, and, for TCSC courses, $\rho=0$. 


\section{References}

1. Dingle, H. \& Drake, V. A. What Is Migration? BioScience 57, 113-121 (2007).

2. Mouritsen, H. Long-distance navigation and magnetoreception in migratory animals. Nature 558, 50-59 (2018).

3. Able, K. P. The concepts and terminology of bird navigation. Journal of Avian Biology 32, 174-183 (2001).

4. Berdahl, A. M. et al. Collective animal navigation and migratory culture: from theoretical models to empirical evidence. Phil. Trans. R. Soc. B 373, 20170009 (2018).

5. Johnston, S. T. \& Painter, K. J. Modelling collective navigation via non-local communication. 13.

6. Wiltschko, R. \& Wiltschko, W. Avian Navigation: A Combination of Innate and Learned Mechanisms. in Advances in the Study of Behavior vol. 47 229-310 (Elsevier, 2015).

7. Chernetsov, N. Compass systems. J Comp Physiol A 203, 447-453 (2017).

8. Sjöberg, S. \& Muheim, R. A New View on an Old Debate: Type of Cue-Conflict Manipulation and Availability of Stars Can Explain the Discrepancies between Cue-Calibration Experiments with Migratory Songbirds. Front. Behav. Neurosci. 10, (2016).

9. Emlen, S. T. Migration: Orientation and Navigation. in Avian Biology 129-219 (Elsevier, 1975). doi:10.1016/B978-0-12-249405-5.50011-2.

10. Muheim, R., Philips, J. B. \& Åkesson, S. Polarized Light Cues Underlie Compass Calibration in Migratory Songbirds. Science 313, 837-839 (2006).

11. Muheim, R., Schmaljohann, H. \& Alerstam, T. Feasibility of sun and magnetic compass mechanisms in avian long-distance migration. Mov Ecol 6, 8 (2018).

12. Reppert, S. M. \& de Roode, J. C. Demystifying Monarch Butterfly Migration. Current Biology 28, R1009R1022 (2018).

13. Kiepenheuer, J. The magnetic compass mechanism of birds and its possible association with the shifting course directions of migrants. Behav Ecol Sociobiol 14, 81-99 (1984).

14. Alerstam, T. \& Pettersson, S.-G. Orientation along great circles by migrating birds using a sun compass. Journal of Theoretical Biology 152, 191-202 (1991).

15. Alerstam, T., Gudmundsson, G. A., Green, M. \& Hedenström, A. Migration Along Orthodromic Sun Compass Routes by Arctic Birds. Science 291, 300-303 (2001). 
16. Mouritsen, H. \& Mouritsen, O. A Mathematical Expectation Model for Bird Navigation based on the Clockand-Compass Strategy. Journal of Theoretical Biology 207, 283-291 (2000).

17. McLaren, J. D., Shamoun-Baranes, J. \& Bouten, W. Wind selectivity and partial compensation for wind drift among nocturnally migrating passerines. Behavioral Ecology 23, 1089-1101 (2012).

18. Alerstam, T. Conflicting Evidence About Long-Distance Animal Navigation. Science 313, 791-794 (2006).

19. Åkesson, S. \& Bianco, G. Route simulations, compass mechanisms and long-distance migration flights in birds. J Comp Physiol A 203, 475-490 (2017).

20. Thorup, K. et al. Flying on their own wings: young and adult cuckoos respond similarly to long-distance displacement during migration. Sci Rep 10, 7698 (2020).

21. Fitzgerald, T. M. \& Taylor, P. D. Migratory orientation of juvenile yellow-rumped warblers (Dendroica coronata) following stopover: sources of variation and the importance of geographic origins. Behav Ecol Sociobiol 62, 1499-1508 (2008).

22. Thorup, K. et al. Juvenile Songbirds Compensate for Displacement to Oceanic Islands during Autumn Migration. PLoS ONE 6, e17903 (2011).

23. Pepper, J. V. Harriot's calculation of the meridional parts as logarithmic tangents. Arch. Rational Mech. 4, 359413 (1968).

24. Alerstam, Thomas. Bird Migration Performance on the Basis of Flight Mechanics and Trigonometry. in Biomechanics in animal behaviour (eds. P. Domenici \& R.W. Blake) (Oxford University Press, 2000).

25. Kendall, D. G. Pole-Seeking Brownian Motion and Bird Navigation. Journal of the Royal Statistical Society. Series B (Methodological) 36, 365-417 (1974).

26. Simons, A. Many wrongs: the advantage of group navigation. Trends in Ecology \& Evolution 19, 453-455 (2004).

27. Thébault, E. et al. International Geomagnetic Reference Field: the 12th generation. Earth Planet Sp 67, 79 (2015).

25 28. Guilford, T. \& Taylor, G. K. The sun compass revisited. Animal Behaviour 97, 135-143 (2014).

29. Mouritsen, H. \& Larsen, O. Migrating songbirds tested in computer-controlled Emlen funnels use stellar cues for a time-independent compass. The Journal of experimental biology 204, 3855-65 (2001). 
30. Mardia, K. V. Statistics of Directional Data. Journal of the Royal Statistical Society. Series B (Methodological) 37, 349-393 (1975).

31. Bäckman, J. \& Alerstam, T. Orientation scatter of free-flying nocturnal passerine migrants: components and causes. Animal Behaviour 65, 987-996 (2003).

32. Zylstra, E. R. et al. Changes in climate drive recent monarch butterfly dynamics. Nat Ecol Evol 5, 1441-1452 (2021).

33. Sim, I. M. W., Green, M., Rebecca, G. W. \& Burgess, M. D. Geolocators reveal new insights into Ring Ouzel Turdus torquatus migration routes and non-breeding areas. Bird Study 62, 561-565 (2015).

34. Lisovski, S. et al. The Indo-European flyway: Opportunities and constraints reflected by Common Rosefinches breeding across Europe. J Biogeogr 48, 1255-1266 (2021).

35. Thorup, K. \& Rabøl, J. The orientation system and migration pattern of long-distance migrants: conflict between model predictions and observed patterns. Journal of Avian Biology 32, 111-119 (2001).

36. Cooper, N. W., Hallworth, M. T. \& Marra, P. P. Light-level geolocation reveals wintering distribution, migration routes, and primary stopover locations of an endangered long-distance migratory songbird. $J$ Avian Biol 48, 209-219 (2017).

37. Kruszynski, C. et al. Identifying migratory pathways of Nathusius' pipistrelles ( Pipistrellus nathusii) using stable hydrogen and strontium isotopes. Rapid Commun Mass Spectrom 35, (2021).

38. Sokolovskis, K. et al. Ten grams and 13,000 km on the wing - route choice in willow warblers Phylloscopus trochilus yakutensis migrating from Far East Russia to East Africa. Mov Ecol 6, 20 (2018).

39. del Hoyo, J. \& Collar, N. J. Handbook of the Birds of the World and BirdLife International digital checklist of the birds of the world. vol. 1 (2016).

40. Bächler, E. et al. Year-Round Tracking of Small Trans-Saharan Migrants Using Light-Level Geolocators. PLoS ONE 5, e9566 (2010).

41. Gómez, C. et al. Fuel loads acquired at a stopover site influence the pace of intercontinental migration in a boreal songbird. Sci Rep 7, 3405 (2017).

42. Åkesson, S., Morin, J., Muheim, R. \& Ottosson, U. Dramatic Orientation Shift of White-Crowned Sparrows Displaced across Longitudes in the High Arctic. Current Biology 15, 1591-1597 (2005). 
43. Lefeldt, N., Dreyer, D., Schneider, N.-L., Steenken, F. \& Mouritsen, H. Migratory blackcaps tested in Emlen funnels can orient at 85 degrees but not at 88 degrees magnetic inclination. Journal of Experimental Biology 218, 206-211 (2015).

44. Alerstam, T. et al. Great-circle migration of Arctic passerines. Auk 125, 831-838 (2008).

45. Thorup, K. \& Rabøl, J. Compensatory behaviour after displacement in migratory birds: A meta-analysis of cage experiments. Behav Ecol Sociobiol 61, 825-841 (2007).

46. Rabol, J. The Orientation Systems of Long-Distance Passerine Migrants Displaced in Autumn from Denmark to Kenya. Ornis Scandinavica 24, 183 (1993).

47. Mouritsen, H. et al. An experimental displacement and over 50 years of tag-recoveries show that monarch butterflies are not true navigators. Proceedings of the National Academy of Sciences 110, 7348-7353 (2013).

48. Shamoun-Baranes, J., Liechti, F. \& Vansteelant, W. M. G. Atmospheric conditions create freeways, detours and tailbacks for migrating birds. J Comp Physiol A 203, 509-529 (2017).

49. Lindecke, O., Elksne, A., Holland, R. A., Pētersons, G. \& Voigt, C. C. Experienced Migratory Bats Integrate the Sun's Position at Dusk for Navigation at Night. Current Biology 29, 1369-1373.e3 (2019).

50. Thorup, K., Rabøl, J. \& Erni, B. Estimating variation among individuals in migration direction. Journal of Avian Biology 38, 182-189 (2007).

51. Hongre, L., Sailhac, P., Alexandrescu, M. \& Dubois, J. Nonlinear and multifractal approaches of the geomagnetic field. Physics of the Earth and Planetary Interiors 110, 157-190 (1999).

52. Gill, J. A., Alves, J. A. \& Gunnarsson, T. G. Mechanisms driving phenological and range change in migratory species. Phil. Trans. R. Soc. B 374, 20180047 (2019).

53. Kays, R., Crofoot, M. C., Jetz, W. \& Wikelski, M. Terrestrial animal tracking as an eye on life and planet. Science 348, aaa2478-aaa2478 (2015).

54. Gade, K. A Non-singular Horizontal Position Representation. The Journal of Navigation 63, 395-417 (2010).

55. Brett, A. C. \& Tuller, S. E. The Autocorrelation of Hourly Wind Speed Observations. Journal of Applied Meteorology (1988-2005) 30, 823-833 (1991).

56. Aurbach, A., Schmid, B., Liechti, F., Chokani, N. \& Abhari, R. Complex behaviour in complex terrain Modelling bird migration in a high resolution wind field across mountainous terrain to simulate observed patterns. Journal of Theoretical Biology 454, 126-138 (2018). 
57. Schmidt-Koenig, K. The sun compass. Experientia 46, 336-342 (1990).

58. Hoinville, T. \& Wehner, R. Optimal multiguidance integration in insect navigation. Proc Natl Acad Sci USA 115, 2824-2829 (2018).

59. Hillen, T., J. Painter, K., C. Swan, A. \& D. Murtha, A. Moments of von mises and fisher distributions and applications. Mathematical Biosciences and Engineering 14, 673-694 (2017).

60. Laundal, K. M. \& Richmond, A. D. Magnetic Coordinate Systems. Space Sci Rev 206, 27-59 (2017).

61. Wehner, R. \& Muller, M. The significance of direct sunlight and polarized skylight in the ant's celestial system of navigation. Proceedings of the National Academy of Sciences 103, 12575-12579 (2006).

62. Alerstam, T. \& Lindström, Å. Optimal Bird Migration: The Relative Importance of Time, Energy, and Safety. in Bird Migration (ed. Gwinner, E.) 331-351 (Springer, 1990). doi:10.1007/978-3-642-74542-3_22.

63. McLaren, J. D., Shamoun-Baranes, J. \& Bouten, W. Stop early to travel fast: modelling risk-averse scheduling among nocturnally migrating birds. Journal of Theoretical Biology 316, 90-98 (2013).

64. Burnham, K. P., Anderson, D. R. \& Huyvaert, K. P. AIC model selection and multimodel inference in behavioral ecology: some background, observations, and comparisons. Behav Ecol Sociobiol 65, 23-35 (2011).

Acknowledgments: We thank Henrik Mouritsen and Michael Winklhofer for fruitful discussions.

Funding: German Research Foundation SFB 1372 "Magnetoreception and navigation in vertebrates" INST 184/205-1 (BB, HS)

Author contributions: All authors worked to conceive the study. J.D.M. formulated and coded the models, analysed and visualized the results, wrote draft manuscript, and led manuscript revisions. H.S. and B.B. supervised the study, helped administer the project, and helped with manuscript revisions. 


\section{Supplementary Files}

This is a list of supplementary files associated with this preprint. Click to download.

- McLarenetalSuppllnfocombined.docx 